Ebisu Ebisu

Études japonaises Études japonaises

$58 \mid 2021$

2011-2021 : crises, ruptures et nouvelles dynamiques.

Dix ans après la triple catastrophe du 11 mars

\title{
Résilience des transports face à la catastrophe : séisme et tsunami du 11 mars 2011 au Japon \\ 炎害における交通のレジリエンス: 東日本大震炎化书ける地震と津 \\ 波
}

Transportation Resilience in Times of Disaster: 2011 Earthquake and Tsunami in Japan

Léo Martial, Fumihiko Nakamura, Itsuki Yoshida et Riko Sakuda

\section{OpenEdition}

Journals

Édition électronique

URL : https://journals.openedition.org/ebisu/5683

DOI : 10.4000/ebisu.5683

ISSN : 2189-1893

Éditeur

Institut français de recherche sur le Japon à la Maison franco-japonaise (UMIFRE 19 MEAE-CNRS)

Édition imprimée

Date de publication : 1 novembre 2021

Pagination : 47-92

ISSN : 1340-3656

Référence électronique

Léo Martial, Fumihiko Nakamura, Itsuki Yoshida et Riko Sakuda, « Résilience des transports face à la catastrophe : séisme et tsunami du 11 mars 2011 au Japon », Ebisu [En ligne], 58 | 2021, mis en ligne le 01 novembre 2021, consulté le 13 avril 2022. URL : http://journals.openedition.org/ebisu/5683 DOI : https://doi.org/10.4000/ebisu.5683 


\title{
Résilience des transports face à la catastrophe : séisme et tsunami du 11 mars 2011 au Japon Léo Martial, Nakamura Fumihiko, Yoshida Itsuki, Sakuda Riko
}

\author{
災害における交通のレジリエンス：東日本大震災における地震と津波 \\ レオ・マーシャル、中村文彦、吉田樹、作田莉子
}

Transportation Resilience in Times of Disaster:

2011 Earthquake and Tsunami in Japan

Léo Martial, NaKamura Fumihiko, Yoshida Itsuki, SaKuda Riko

\begin{abstract}
\ots-clés : énergie, plan d'urgence, planification, résilience, transports

Les auteurs : Léo Martial est docteur de l'Institut d'innovation urbaine de l'université nationale de Yokohama, où Fumihiko Nakamura est professeur et Riko Sakuda étudiante en master. Itsuki Yoshida est maître de conférences à la faculté d'économie et d'administration des affaires de l'université de Fukushima.
\end{abstract}

Résumé : Le séisme de la côte Pacifique du Tōhoku survenu le 11 mars 2011 a provoqué des interruptions majeures dans les réseaux de transports, fragilisant soudainement une grande partie du territoire japonais et poussant les autorités à réagir rapidement. Les réfugiés et les habitants des zones sinistrées ont dû adapter leurs besoins en mobilité. Cette situation inédite a démontré la nécessité d'accroître la résilience des infrastructures de transport japonaises afin d'améliorer sensiblement les capacités de reconstruction matérielle et sociale lors d'une situation d'urgence. Cet article fournit une analyse des dégâts et impacts de la catastrophe sur les transports et leur infrastructure énergétique, un examen des mesures adoptées et une évaluation de leurs capacités passées et acquises de résilience. 
】キーワード

エネルギー、エマージェンシープランニング、

計画化、レジリエンス、交通

\section{著者}

レオ・マーシャルは、横浜国立大学都市イノ ベーション研究院の博士。同研究所では、中村 文彦氏が教授を務め作田莉子が修士課程に在 籍する。吉田樹は福島大学経済経営学類准教授。

要旨

2011 年 3 月 11 日に発生した東日本大震災は、
交通網に大きな混乱をもたらし、日本の多く の地域を突如として脆弱化させ、当局は迅速 な対応を迫られた。避難民や被災地の住民は、 移動手段を変えなければならなかった。この 未曾有の事態は、緊急時の物理的・社会的な 復興能力を大幅に向上させるため、日本の交 通インフラの回復力を高める必要性を示した。 本稿では、震災による交通・エネルギーイン フラの被害と影響の分析や対策の検討、過去 と現在の交通の回復力の評価を行う。
خ Keywords: Energy, Emergency

Planning, Planning, Resilience, Transport

The Authors: Léo Martial is a PhD graduate from the Institute of Urban Innovation at Yokohama National University, where Fumihiko Nakamura is a professor and Riko Sakuda is a master's graduate. Itsuki Yoshida is an associate professor at the Faculty of Economics and Business Administration, Fukushima University.

Abstract: The Tōhoku Pacific Coast earthquake on 11 March 2011 severely disrupted transport networks, suddenly jeopardising a large part of Japan, and prompting authorities to take fast action. Refugees and inhabitants of the affected areas were forced to adapt their transport needs. This unprecedented situation laid bare the need for a more resilient Japanese transportation infrastructure in order to significantly improve physical and social reconstruction capacity during an emergency situation. This paper analyses the damage and impact of the disaster on transport and its energy infrastructure, reviews the measures adopted, and assesses past and acquired resilience. 


\title{
Résilience des transports face à la catastrophe : séisme et tsunami du 11 mars 2011 au Japon
}

\author{
Léo Martial ${ }^{*}$, Nakamura Fumihiko**, Yoshida Itsuki*** \& Sakuda Riko****
}

\section{Introduction}

Les sociétés contemporaines dépendent de plus en plus des infrastructures énergétiques, des transports et des télécommunications. Ces services essentiels à la vie quotidienne sont devenus au cours des dernières décennies techniquement complexes, interdépendants et onéreux, les rendant vulnérables et difficiles à maintenir. Cette dépendance, toujours plus grande, peut entraîner lors de situations inattendues des pertes humaines et financières considérables, les systèmes de transport étant souvent parmi les plus affectés par les catastrophes naturelles, bien qu'ils demeurent essentiels au secours des sinistrés dans les zones touchées et au soutien des interventions d'urgence et de rétablissement à long terme (Graham et al. 2007).

Plusieurs concepts ont été proposés afin d'étudier la performance des systèmes de transport lors d'une exposition à diverses perturbations, allant des fluctuations quotidiennes fréquentes aux catastrophes naturelles rares; le terme résilience apparaît de plus en plus dans la littérature scientifique. En comparaison d'autres notions comme la robustesse, la fiabilité, la capacité

* Docteur à l'Institut d'innovation urbaine, université nationale de Yokohama.

** Professeur à l'Institut d'innovation urbaine, université nationale de Yokohama.

*** Professeur associé à la faculté d'économie et d'administration des affaires, Fukushima Future Center for Regional Revitalization (FURE), université de Fukushima.

****Masterante à l'Institut d'innovation urbaine, université nationale de Yokohama. 
de survie et la flexibilité, la résilience se concentre sur la réduction et la récupération des performances face à des perturbations inévitables. Dans la conception d'un système, on doit considérer et "absorber" les variations quotidiennes pour ainsi maintenir ses fonctions. Les séismes et autres catastrophes de grande ampleur écartent fatidiquement le système de son état d'équilibre. La capacité d'un système à réduire efficacement à la fois l'amplitude et la durée de l'écart par rapport aux niveaux de performance planifiés est la définition la plus significative de la résilience. En effet, si la résilience qualifiait originellement la résistance d'un matériau aux chocs, sa définition a ensuite été étendue à la capacité d'un système, d'un organisme, d'une espèce ou d'une structure à surmonter une détérioration de son environnement. D'abord défini dans le contexte des systèmes écologiques en établissant une distinction entre résilience et stabilité (Holling 1973), le concept a par la suite été introduit dans différentes disciplines comme l'organisation (Sheffi 2015), l'économie (Rose 2014), les sciences sociales (Adger 2000), les chaînes d'approvisionnement (Christopher 2004) ou l'ingénierie (Hollnagel 2006). La notion de résilience accepte plusieurs interprétations selon le domaine, mais la plupart partage cette idée de la capacité d'un système à revenir à son état initial après de lourdes perturbations en changeant profondément la structure (Hosseini 2016). La résilience a largement été étudiée en ingénierie des transports, en particulier ces dernières années. Ces études se concentrent notamment sur les caractéristiques intrinsèques des réseaux physiques, mais peu mesurent la résilience des nœuds d'échange, de l'acheminement énergétique et des répercussions économiques et sociales (Reghezza-Zitt et al. 2015). Aussi, les méthodes d'évaluation de la résilience des principaux pôles de transport aux échelles locales ou nationales font particulièrement défaut.

Le Japon a mis en place de nombreuses institutions et législations sur la base des leçons tirées à la suite des catastrophes naturelles survenues depuis l'après-guerre. Les interventions d'urgence telles que la fourniture de biens de secours, la gestion des abris d'évacuation et la construction d'abris de transition, sont menées conformément à la saigai kyūjo hō 災害 救助法 (loi sur les secours en cas de catastrophe) dont la gestion est assurée par le ministère de la Santé, du Travail et des Affaires sociales (Kōsei rōdōshō 厚生労働省, MHLW). Les infrastructures publiques sont, quant à elles, réhabilitées conformément à trois lois dont l'application est respectivement vérifiée par : 
- le ministère de l'Aménagement du territoire, des Infrastructures, des Transports et du Tourisme (Kokudo kōtsūshō 国土交通省, MLIT) pour la reconstruction des ouvrages publics de génie civil endommagés par la catastrophe;

- le ministère de l'Agriculture, des Forêts et de la Pêche (Nōrin suisanshō 農林水産省, MAFF) pour la reconstruction des installations agricoles, forestières et halieutiques;

- le ministère de l'Éducation, de la Culture, des Sports, des Sciences et de la Technologie (Monbu kagakushō 文部科学省, MEXT) pour la reconstruction des installations scolaires.

En cas de catastrophe majeure, les gouvernements locaux peuvent également bénéficier d'un soutien financier spécial. Toutefois, si les programmes de réhabilitation des grandes infrastructures publiques s'avèrent efficaces, les mécanismes de soutien aux populations touchées, mis en place en 1999 à la suite du grand tremblement de terre de Hanshin-Awaji en 1995 à Kobe, sont jugés souvent lents et insuffisants en comparaison, car ils ne fournissent qu'une aide limitée dans la reprise économique et sociale des individus.

Au-delà des nombreuses victimes, les conséquences du séisme et du tsunami de la côte Pacifique du Tōhoku survenus le 11 mars 2011 ont été désastreuses pour les infrastructures de transport, dont de nombreuses essentielles à la survie des populations et indispensables aux interventions d'urgence ont été détruites ou fortement endommagées. Les zones affectées ont été si vastes que les agences responsables des interventions d'urgence, du soutien logistique et de la protection civile ont subi des pertes et des pénuries de ressources inédites, réduisant considérablement leur opérabilité, voire dans certains cas provoquant leur interruption totale. La situation était critique du fait de l'effondrement de ponts et viaducs, des voies obstruées par des fissures dans leur revêtement, par des glissements de terrain ou par des débris charriés par le tsunami, de sorte que des centaines de sections d'autoroutes et de routes nationales ont été fermées. En raison d'une extrême pénurie de carburant dans plusieurs départements et afin de faciliter les opérations de sauvetage, les autorités ont immédiatement exhorté la population à éviter d'utiliser les véhicules particuliers. Le service du train à grande vitesse Tōhoku Shinkansen ne reprit entièrement qu'après 49 jours, tandis que la remise en service des lignes de chemin de fer conventionnelles du Tōhoku a demandé, dans certains cas, des mois voire des années de 
travaux. L'aéroport de Sendai ainsi que de nombreuses installations portuaires ont été également lourdement touchés; les télécommunications ont été limitées, voire interrompues.

L’amélioration de la résilience des infrastructures énergétiques et de transport est débattue depuis plusieurs années, et cet article propose donc une approche originale en la mettant en perspective au moyen de l'analyse d'une situation catastrophique à l'ampleur inédite. La première partie fournit une analyse des dégâts et des conséquences du séisme et du tsunami du 11 mars 2011, un examen des mesures adoptées par différents ministères durant trois phases distinctes - urgence, transition, reprise - et une évaluation des capacités passées et acquises de résilience des infrastructures de transports. La deuxième partie examine les conséquences de la catastrophe sur l'approvisionnement énergétique des transports. La troisième étudie la situation de quatre réseaux de transports : routier, ferroviaire, maritime et aérien, ainsi que celle des réseaux de télécommunication. La quatrième et dernière partie présente des axes de recherches potentiels sur la résilience des transports à plus long terme dans l'archipel japonais : elle revient pour cela sur les leçons tirées en établissant un parallèle entre catastrophe destructrice sur le court terme et résilience sur le long terme.

\section{La question énergétique}

Le séisme et le tsunami du 11 mars ainsi que l'accident nucléaire de Fukushima ont animé le débat énergétique dans l'Archipel et placé au centre des discussions les termes " changement ", "communauté ", "leadership ", "vulnérabilité " et " résilience " (Samuels 2013). La perception de l'énergie pour les citoyens passe d'une simple commodité du quotidien à un aperçu d'un système hautement complexe, parfois conflictuel, défaillant ou biaisé. Le nucléaire a été au cœur du débat pour les changements de politique de production d'électricité (Aoki et al. 2013), mais la catastrophe a également mis en valeur la dépendance automobile et pétrolière notamment dans les zones périurbaines et rurales. 


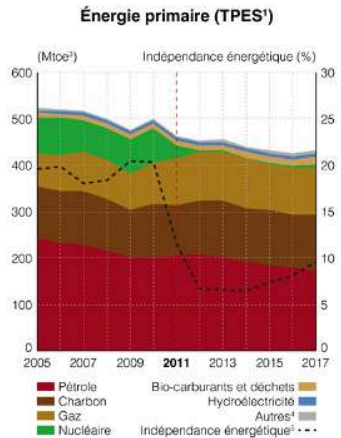

Carburants

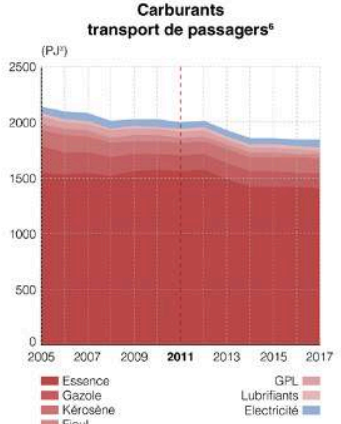

Production nationale d'électricité

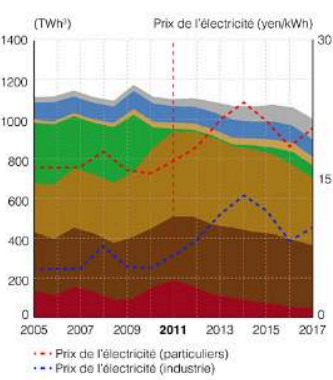

Carburants

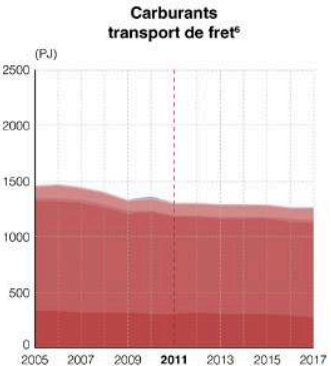

Consommation finale ( $\left.\mathrm{TFC}^{2}\right)$ par secteur

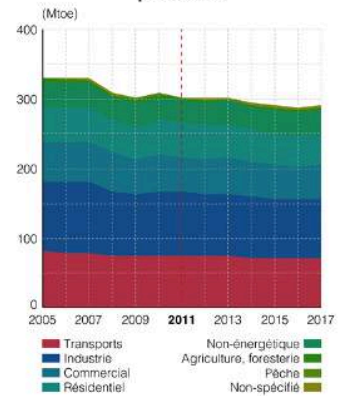

Consommation d'énergie

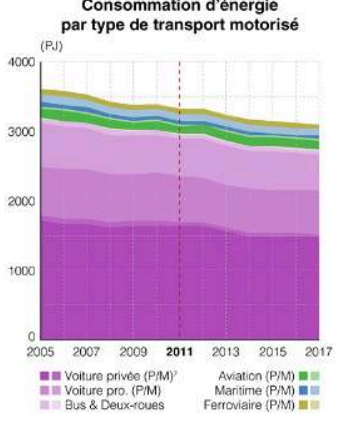

1. Total Primary Enorgy Supply: Consommation totaio d'énorgio primairo, avant pertes of transformations

2. Total Finat Consumption : Consommation d'energie tinale, apros pertes ef transtomations (TFC Japon, 2010:62,21\% TPES, 2017:67.77 \% TPES)

3. Torne équivalent pótrole $(1 \mathrm{Mios}=41,868 \mathrm{PJ}=11,630 \mathrm{TWh})$

4. Photovoitaique, géothermie, efolien, biomasse, déchets municipaux, autres dechiets

5. Rapport entre production et consommation nationales d'énergie primaive

6. Charbon et gaz naturel négiligleables, non-représentés

7. $P$ = Passagers, $M=$ Marchandises

\section{Fig. 01}

Évolution de la situation énergétique du Japon depuis la triple catastrophe du 11 mars 2011. Sources: Agence Internationale de l'énergie, METI.

Réalisation : Martial (2021). 


\section{1. Acheminement des produits pétroliers}

En 2010, les transports - notamment routier, aérien et maritime, qui dépendent quasi intégralement du pétrole sous plusieurs de ses formes : essence, kérosène, gazole, fioul lourd - ont consommé 24,3\% de l'énergie finale de l'Archipel et ont fonctionné avec $97 \%$ de produits pétroliers ${ }^{1}$. Seul le transport ferroviaire, presque entièrement électrifié au Japon ${ }^{2}$, peut recourir massivement à plusieurs formes d'énergie pour la production d'électricité. Les infrastructures pétrolières sont donc vitales pour une grande partie du transport de voyageurs et de marchandises.

Le Japon ne dispose que de ressources pétrolières négligeables sur son territoire et se retrouve tributaire des importations. Différents groupes - publics comme privés - conservent donc des stocks stratégiques importants pour se préserver des conséquences d'une interruption soudaine de leur approvisionnement. Toutefois, le pays connaît une diminution régulière de sa consommation de pétrole depuis 2005 en raison d'une baisse et d'un vieillissement de sa population, d'une érosion industrielle, d'une substitution lente mais continue des carburants et d'objectifs d'efficacité énergétique plus drastiques (Granier 2019). Aussi, le gaz naturel tend à remplacer le pétrole dans certains secteurs industriels; le chauffage domestique et commercial s'électrifie progressivement. La fermeture des centrales nucléaires à la suite du séisme de 2011 a néanmoins contraint le Japon à accroître ses importations de charbon et de pétrole brut pour combustion directe dans ses centrales électriques, augmentant leurs facteurs de charge ${ }^{3}$.

Le séisme et le tsunami du 11 mars 2011 ont ainsi engendré une grave pénurie de pétrole, affectant de manière significative les transports et la logistique dans une majeure partie des régions du Tōhoku et du Kantō. Le transport routier est devenu difficile du fait de l'épuisement des réserves de

1. Enerugī hakusho 2012, dai 2 bu enerugī dōkō 1 エネルギー白書2012、第2部 エネルギー 動向1 (Livre blanc sur l'énergie 2012, volume 1, chapitre 2, tendances énergétiques, METI).

2. Selon l'Ippan shadanhōjin nippontetsudō sharyō kōgyōkai 一般社団法人日本鉄道車 輌工業会 (Association industrielle japonaise des véhicules ferroviaires). Les locomotives diesel produites ne représentaient que 2,8 \% du marché japonais en 2010 : https://www. tetsushako.or.jp/data.html (dernière consultation le 26 juillet 2020).

3. Rapport entre la puissance moyenne effective d'une centrale électrique sur une période donnée et sa puissance nominale. 
carburant dans de nombreuses stations-service. Les stations restantes ont dû drastiquement rationner leurs stocks respectifs devant la forte demande, occasionnant de longues files d'attente ${ }^{4}$. Cette situation s'est poursuivie pendant plusieurs semaines dans différentes zones du Tōhoku, notamment dans les départements de Miyagi et d'Iwate qui se trouvaient en situation encore critique trois semaines après la catastrophe. Ces manques en carburant ont gravement affecté de nombreuses activités : de grandes difficultés dans les opérations de sauvetage et l'évacuation de la plupart des milliers de réfugiés dans certaines zones, entraves majeures à la livraison de fournitures d'urgence dans les zones touchées, baisse voire suspension de nombreuses activités socioéconomiques, déclin des fonctions logistiques, rupture des chaînes d'approvisionnement industrielles et manufacturières, le tout engendrant des pertes économiques considérables, chiffrables en billions de yens (Kajitani et al. 2013). Ce fut la pénurie la plus importante dans l'Archipel depuis les crises pétrolières des années 1970.

Les premières informations rendues accessibles au public pour illustrer de manière globale la pénurie de pétrole ont été insuffisantes. Si le ministère de l'Économie, du Commerce et de l'Industrie (Keizai sangyōshō 経済産 業省, METI) a commencé à publier en ligne des informations concernant les mesures et opérations menées seulement une semaine après le séisme, ni le METI ni la Petroleum Association of Japan (PAJ) n'ont communiqué de résultats d'analyses permettant une compréhension quantitative globale de la situation. Le gouvernement et l'industrie pétrolière ont tout d'abord attribué la pénurie aux raffineries et aux installations portuaires endommagées. Les trois raffineries du Tōhoku touchées représentaient en 2011 un dixième de la capacité nationale de raffinage de 4,5 millions de barils par jour. Cependant, le facteur de charge standard d'une raffinerie se situant autour de $80 \%$, la capacité nationale de raffinage peut rapidement retrouver un équilibre en augmentant la production de vingt-et-un autres sites restés en service ${ }^{5}$, mais la localisation de ces dernières, dans les régions

4. Média spécialiste des véhicules motorisés au Japon Resuponsuレスポンス (Response) : https://response.jp/article/2011/04/28/155691.html.

5. "Seiyusho no shozaichi to gen.yu shori nōryoku (2011-nen 1 gatsugenzai)" 製油所の所在地と原油処理能力 (2011年1月現在) (Emplacements des raffineries et capacité de traitement du pétrole brut en janvier 2011) : https://www.paj.gr.jp/from_chairman/data/20110317_g.pdf (dernière consultation le 26 juillet 2020). 
lointaines du Kansai et du Chūgoku, rend difficile un acheminement vers le Kantō et le Tōhoku. Trois autres raffineries du Kantō ont connu un arrêt d'exploitation durant dix jours, une explosion dans celle de Chiba causant six blessés. Cette insuffisance d'informations de la part du METI et de la PAJ laissa également plusieurs questions en suspens sur les mesures mises en œuvre, leurs résultats et les raisons de la durée de la pénurie : près d'un mois dans certaines zones. La thèse d'un accaparement et d'un stockage du pétrole par certains consommateurs a été rapidement écartée en raison de la mauvaise interprétation des faits qu'elle apportait (Akamatsu et al. 2016). Des études ultérieures indépendantes ont révélé que la quantité de produits pétroliers fournis à la région du Tōhoku n’a pas suffi, son approvisionnement renforcé depuis Hokkaidō n’ayant pas entièrement compensé la baisse des livraisons depuis le Kantō. Les livraisons importantes depuis l'Ouest du Japon annoncées par le METI, notamment depuis les régions du Kansai et du Chūgoku, n’ont en réalité représenté qu’un dixième des déclarations du ministère. Le volume transporté vers le Tōhoku correspondait finalement à environ un tiers de la demande normale au cours des deux semaines qui ont suivi la catastrophe. Au cours de ce laps de temps, dans le Tōhoku, seuls les ports de la mer du Japon étaient en état de fonctionnement. Les ports des zones touchées de la côte Pacifique n’ont retrouvé une opérabilité partielle qu'à partir du 23 mars, accueillant alors les premiers pétroliers et soulageant ainsi la pénurie. En termes d'offre, une semaine après la catastrophe, de nombreuses municipalités des départements d'Akita et de Yamagata - sur le littoral de la mer du Japon - ont pu conserver un taux d'approvisionnement de plus de $60 \%$ (néanmoins inférieur aux premiers jours suivant la catastrophe) mais presque aucun carburant n'a pu être distribué sur la côte Pacifique. Après deux semaines, le taux d'approvisionnement est remonté à plus de $80 \%$ dans la plupart des municipalités sur la côte de la mer du Japon, mais de graves pénuries se sont poursuivies sur la côte Pacifique. Au bout de trois semaines, tandis que l'offre et la demande côté mer du Japon étaient presque revenues à la normale, le taux d'approvisionnement demeurait souvent inférieur à $40 \%$ dans certaines municipalités le long de la côte Pacifique, où la pénurie de carburant n'était toujours pas résolue un mois après la catastrophe (fig. 03). Iwate et Miyagi ont par ailleurs représenté $80 \%$ d'une importante disparition de la demande, reflétant le ralentissement économique de la région. Cette disparition a pu représenter 
dans certaines municipalités de Miyagi la moitié de la consommation en comparaison du mois de mars 2010.

Cette situation critique a rappelé la nature indispensable du pétrole dans les systèmes de transport contemporains. Le pétrole est un bien d'utilité publique soutenant les activités socioéconomiques et la suspension de sa fourniture à court terme entraîne d'énormes pertes économiques. En effet, si le coût requis pour le transfert exceptionnel d'essence depuis d'autres régions de l'Archipel a été estimé entre 200 et 300 millions de yens, les pertes économiques ont, quant à elles, été estimées entre 150 et 200 milliards de yens (Akamatsu et al. 2016). L'expérience et les connaissances acquises lors de cet événement devraient être pleinement utilisées pour développer de nouvelles méthodes préventives contre de futures catastrophes, afin d'éviter que cette situation ne se reproduise lors de nouveaux tremblements de terre sur les failles de Tōkai, Tōnankai ou Nankai à proximité des plus grandes villes japonaises. Ces mesures préventives pourraient inclure le renforcement des installations fournissant les produits pétroliers, une augmentation des stocks ou la conception d'un système national de soutien en cas de séisme. Une planification renforcée des stratégies logistiques pour fournir des produits pétroliers en urgence dans des domaines spécifiques semble souhaitable. De nombreuses industries ont depuis affiné leurs plans de continuité des affaires leur permettant de fonctionner en mode dégradé 6 lors d'un désastre.

6. Situation où tout ou partie d'une entreprise ou d'un autre groupe doit fonctionner sans ses ressources humaines et matérielles habituelles. 


\section{2. Approvisionnement électrique}

Lélectricité ne représentait en 2011 que 3,1\% des besoins énergétiques des transports de passagers au Japon et était quasi complètement consommée par le ferroviaire. Si sa consommation augmente, sa proportion baisse en comparaison des 7,2 \% de 1965, avant la grande motorisation de l'Archi$\mathrm{pel}^{7}$. Cette faible proportion ne reflète néanmoins pas l'importance du chemin de fer au Japon. Entièrement électrifié à de rares exceptions pour des itinéraires côtiers ou touristiques ${ }^{8}$, énergétiquement efficient, le train représente le tiers du volume national annuel de transport de passagers ${ }^{9}$. La mise hors service de nombreuses centrales thermiques de la côte Pacifique et des centrales nucléaires dans le pays entier a fait peser sur le réseau électrique japonais un risque de black-out majeur. Dans l'aire urbaine de la capitale, la Tokyo Electric Power Company (Tepco) ne pouvant, suite au séisme, délivrer qu'une puissance de $31 \mathrm{GW}$ - contre $52 \mathrm{GW}$ la veille du séisme - pour une demande de 41 GW, le Premier ministre Kan Naoto 菅直人 a approuvé des coupures de courant volontaires touchant 47 millions de personnes, soit $37 \%$ de la population ${ }^{10}$. Si la capacité de production nationale a proportionnellement été moins affectée - en passant de 282 GW à 243 GW il a été impossible d'acheminer suffisamment d'électricité depuis l'Ouest du pays en raison des interconnexions limitées entre ses dix fournisseurs, mais surtout à cause d'une différence de fréquence de l'électricité produite entre Hokkaidō, le Tōhoku, la région de Tokyo d'une part et le reste du pays d'autre part - respectivement $50 \mathrm{~Hz}$ contre $60 \mathrm{~Hz}$ - héritage de la fin du $\mathrm{XIX}^{\mathrm{e}}$ siècle où le réseau électrique japonais s'est développé à partir du Kantō et du Kansai avec des équipements respectivement allemands et américains. La puissance des transformateurs situés dans les départements de Nagano et

7. Voir note 1.

8. Les lignes côtières de Sanriku Railway, abordées ultérieurement, fonctionnent avec des locomotives diesel.

9. Mesure la quantité de transport sous forme de voyageur-kilomètre pour le transport de passagers et de tonne-kilomètre pour le transport de marchandises.

10. Kokka senryakukyoku 国家戦略局 (National Policy Unit) : KKyōkyūryoku no ronten nitsuite » 供給力の論点について (Problèmes d'alimentation électrique) : https://www. cas.go.jp/jp/seisaku/npu/policy09/pdf/20120426/shiryo2.pdf (dernière consultation le 26 juillet 2020). 
Shizuoka n'a été que de 1 GW et représentait moins du dixième de l'acheminement devenu nécessaire après la catastrophe.

Les coupures de courant volontaires ont été destinées à éviter des pannes soudaines pouvant mener à des situations chaotiques. Tepco divise sa zone de services en cinq puis vingt-cinq sections en fonction de l'emplacement des postes de distribution électrique, puis déclenche à tour de rôle des pannes de courant de quelques heures dans chaque section, tout en tentant d'anticiper au mieux l'équilibre entre l'offre et la demande. Les coupures pouvant durer jusqu'à trois heures, certaines lignes de chemin de fer ont été suspendues pendant plusieurs jours dans certaines régions. Cette situation confuse a bloqué un grand nombre de personnes dans les transports ferroviaires, malgré l'anticipation des coupures qui, par ailleurs, étaient les premières depuis 1974 à la suite de la première crise pétrolière ${ }^{11}$.

Sur le plus long terme, la part des combustibles fossiles dans la production énergétique de l'Archipel est passée de $81 \%$ en 2010 à $89 \%$ en 2016, notamment en raison de l'arrêt quasi total de la production d'électricité nucléaire. Le Japon est aujourd'hui le troisième plus gros importateur de charbon et le deuxième de gaz naturel, ce qui affecte grandement sa sécurité énergétique, son économie ${ }^{12}$ et son environnement. Son autosuffisance énergétique, déjà modeste en 2010 avec $20 \%$, s'effondre à 6,7 \% dès 2012 et demeure inférieure à $10 \%$ jusqu'en 2017, plaçant le Japon en avant-dernière place des pays de l'OCDE. Cette hausse des importations de combustibles fossiles a également entraîné une hausse soudaine du prix de l'électricité qui, malgré une tendance à la baisse depuis 2014, demeure sensiblement supérieur en 2017.

Les organismes Denryoku/gasu torihiki kanshi-tō iinkai 電力・ガス取引 監視等委員会 (EGC, de l'anglais Electricity and Gas Market Surveillance Commission) et Denryoku kōiki-teki un.ei suishin kikan 電力広域的 運営推進機関 (OCCTO, de l'anglais Organization for Cross-regional Coordination of Transmission Operators) ont été créés par le METI à

11. Japan for sustainability : https://www.japanfs.org/en/news/archives/news_id030904. html (dernière consultation le 26 juillet 2020).

12. La balance commerciale du Japon, en général largement excédentaire depuis l'aprèsguerre, est devenue négative de 2011 à 2015, le plus gros déficit commercial étant enregistré en 2014. 
l'occasion de la cinquième réforme du marché de l'électricité japonais en 2015. Leur rôle est de promouvoir le développement des réseaux de distribution d'électricité pour garantir son utilisation transrégionale et renforcer ainsi la fonction nationale de maintien de l'équilibre offre-demande d'électricité dans les situations normales et d'urgence. Il est par exemple prévu de tripler la capacité des transformateurs de fréquence à l'horizon 2027, ou encore de doubler la capacité d'échange d'électricité entre Tokyo et le Tōhoku ${ }^{13}$.

Le METI actualisa à plusieurs reprises le enerugī kihon keikakuエネルギー 基本計画 (Plan énergétique de base) avec l'ambition d'un mix énergétique composé de 22 à $24 \%$ d'énergies renouvelables; une autosuffisance de $40 \%$ avec notamment une réduction de la dépendance aux combustibles fossiles à $56 \%$; une réduction de la consommation d'électricité de $17 \%$, le tout d'ici 2030. Pour soutenir ces promesses, le gouvernement collabore avec l'industrie et les universités dans le cadre de promotions d'innovations technologiques et sociétales en matière d'énergie et d'environnement, avec notamment le enerugï/kankyō inobēshon senryaku エネルギー・環境 イノベーション戦略 (NESTI 2050, de l'anglais National Energy and environment Strategy for Technological Innovation towards 2050). Le gouvernement japonais communique fréquemment sur la création d'une infrastructure de production et de distribution d'hydrogène, vecteur énergétique alternatif et potentiellement propre. Les risques d'approvisionnement sont possiblement moindres grâce à de multiples moyens de production et représentent par ailleurs une opportunité pour décarboner la demande d'énergie au Japon, en particulier dans les transports ${ }^{14}$. Contrairement aux accumulateurs, l'hydrogène peut également être stocké à grande échelle et à long terme et rend plus pertinent les sources d'énergie intermittente ${ }^{15}$; il peut ainsi représenter l'une des options de transition énergétique les plus viables

13. Electricity system and market in Japan, METI : https://www.emsc.meti.go.jp/english/info/public/pdf/180122.pdf (dernière consultation le 26 juillet 2020).

14. Basic hydrogen strategy, METI: https://www.meti.go.jp/english/press/2017/ pdf/1226_003b.pdf (dernière consultation le 26 juillet 2020).

15. Grâce à la conversion d'électricité en gaz (Power-to-gas), reposant ici sur l'électrolyse d'eau par de l'électricité pour produire du dihydrogène, le stocker, puis le reconvertir avant de le restituer au réseau électrique. 
dans l'Archipel en regard des défis contemporains comme le pic pétrolier ${ }^{16}$ en approche et le changement climatique.

\section{Les transports}

\section{1. Le réseau routier}

Le réseau routier a été lourdement touché. 540 tronçons de routes à péage, d'autoroutes nationales et départementales ont été fermés. Plus de 400000 véhicules $^{17}, 4198$ routes et 116 ponts ont été détruits ou endommagés, 207 glissements de terrain et 45 ruptures de digues recensés, occasionnant inondations et charriages de déchets sur la voirie, alourdissant ainsi considérablement l'impact sur le réseau. Les routes nationales 6 et 45 reliant respectivement Tokyo à Sendai et Sendai à Aomori par la côte Pacifique ont été particulièrement touchées. Les dommages causés sur la première entraînent l'isolement de nombreuses personnes dans le département de Fukushima; la seconde était un itinéraire auxiliaire important de la Sanriku jidōshadō 三陸自動車道 (autoroute Sanriku), alors incomplète. 16000 personnes se sont trouvées isolées du réseau à la suite du tsunami, notamment dans la péninsule de Funakoshi à Iwate et la ville de Minamisanriku à Miyagi. La péninsule d'Oshika à Miyagi a quant à elle été isolée à la suite de la destruction partielle de la route nationale 398. Quatre heures après le séisme, l’opération de dégagement des routes a été entreprise sous le nom kushi no ha sakusen くしの歯作戦 (opération dents de peigne ${ }^{18}$ ) afin de garantir le plus rapidement possible le passage des véhicules de secours (fig. 01). Cette mission prioritaire a été menée conjointement par les Jieitai 自衛隊 (Forces

16. La production mondiale de pétrole conventionnel, près des trois-quarts de la production totale de pétrole "a franchi un pic en 2008 à 69 millions de barils par jour $(\mathrm{Mb} / \mathrm{j})$, et a décliné depuis d'un peu plus de $2,5 \mathrm{Mb} / \mathrm{j}$ ». L’Agence Internationale de l'Energie estime que ce déclin ne sera pas interrompu (World Energy Outlook 2018, p. 142). Il est probable que le pétrole non-conventionnel ait franchi le pic en 2018.

17. https://www.shinsaihatsu.com/data/110311tsunami_debris.html (dernière consultation le 26 juillet 2020).

18. https://www.soumu.go.jp/soutsu/shikoku/chosa/eisei_inet/pdf/bosai_02.pdf (dernière consultation le 26 juillet 2020). 
japonaises d'autodéfense), la police et différentes organisations départementales et municipales. Elle doit son nom à la création dans un premier temps d'un couloir routier principal composé de l'autoroute Tōhoku malgré plusieurs sections endommagées et de la route nationale 4, qui parcourt aussi l'intérieur du Tōhoku du nord au sud; dans un deuxième temps, douze routes reliant ce couloir jusqu’à la côte Pacifique ont été déblayées en vingt-quatre heures, puis trois autres en deux jours supplémentaires; la troisième et dernière étape a été de déblayer la route nationale 45 de Hachinohe à Sendai - accessible à $97 \%$ après seulement une semaine ${ }^{19}$ - puis la route nationale 6 de Sendai à Iwaki. Un tronçon de plus de $50 \mathrm{~km}$ de cette dernière entre Hirono et Minamisōma a été suspendu en raison des nombreux glissements de terrain et inondations. Après le déblaiement et le retrait des eaux, un tronçon de $20 \mathrm{~km}$ demeure suspendu en raison des retombées radioactives de l'accident de la centrale nucléaire de Fukushima Daiichi 福島第一原子力発電所, qui se situe au milieu de cette portion de route. Dès le 22 avril 2011, seuls les véhicules autorisés ont pu circuler jusqu'au 15 septembre 2014, date de réouverture complète à tous les véhicules ${ }^{20}$. Quelques kilomètres plus au sud, une voie de contournement vers les terres a été inaugurée à Hisanohama le 26 février 2017 afin de diminuer le risque de fermeture en cas de catastrophe et ainsi améliorer la circulation des véhicules d'urgence ${ }^{21}$.

De nombreuses autoroutes sous la juridiction de la East Japan Expressway Company (NEXCO East) ont également été fermées immédiatement après le séisme pour laisser place aux véhicules de secours, puis progressivement rouvertes au public, dès le 24 mars 2011 pour l'autoroute Tōhoku. Les autoroutes de la région ont été accessibles gratuitement aux personnes touchées par la catastrophe jusqu'au 31 mars 2012 afin de soutenir les zones affectées dans leur industrie, leur tourisme, l'évacuation ou le retour de la

19. http://www.thr.mlit.go.jp/road/jisinkannrenjouhou_110311/kokudou/110319_18_kokudou.pdf (dernière consultation le 26 juillet 2020).

20. https://www.minpo.jp/pub/topics/jishin2011/2014/09/post_10676.html (dernière consultation le 26 juillet 2020).

21. http://www.thr.mlit.go.jp/Bumon/kisya/kisyah/images/64190_1.pdf (dernière consultation le 26 juillet 2020). 
population ${ }^{22}$. Les autoroutes et les routes nationales ont été entièrement restaurées fin 2012 à l'exception de certaines zones d'évacuation et de certains ponts de la route nationale 45 . Les dégâts sont restés mineurs dans la zone urbaine de Tokyo, mais un viaduc du réseau autoroutier Shuto a néanmoins connu des dommages importants et plusieurs sections ont été suspendues durant plusieurs semaines ${ }^{23}$. La reconstruction du réseau routier dans les zones touchées a été bien plus rapide et efficace qu'en 1995, où il a fallu plus de dix-huit mois pour totalement réhabiliter celui de Kobe.

L'arrêt de l'exploitation des chemins de fer dans la capitale a provoqué de nombreux embouteillages sur l'ensemble de l'aire métropolitaine, y compris sur les principales artères, mettant en évidence la vulnérabilité du réseau routier. Les très nombreux passagers ferroviaires alors devenus piétons ont en effet rendu la circulation très difficile aux intersections, engendrant selon les données recueillies d'importants gridlocks ${ }^{24}$ (Kiyota et al. 2014). Les premiers embouteillages sont apparus autour du Palais impérial puis ont empiré jusqu'à un blocage généralisé jusqu'aux grandes rocades de l'aire urbaine. 145 intersections bloquées et goulots d'étranglement ont été relevés dans la capitale en comparaison d'une vingtaine seulement lors d'une journée standard. Des embouteillages ont été relevés jusqu'à $16 \mathrm{~h}$ le lendemain dans certaines zones. Plus de 5 millions de personnes ont connu des difficultés pour rentrer à leur domicile le soir de la catastrophe $e^{25}$.

Cette situation chaotique a transformé la bicyclette en seule solution de mobilité fiable pour beaucoup. De nombreux navetteurs n'ont eu d'autre choix que de rentrer chez eux à vélo, souvent sur des distances supérieures à trente kilomètres (Takada et al. 2012). Les ventes

22. NEXCO : «Higashi Nihon daishinsai ni tomonau Tōhoku chihō no kōzokudōro no muryō sochi tsūkō hōhō ni tsuite» 東日本大震災に伴う東北地方の高速道路の無料措 置通行方法について (Méthodes de circulation à voie libre de l'autoroute du district de Tōhoku suite au séisme de 2011 de la côte Pacifique du Tōhoku au Japon).

23. https://www.shutoko.co.jp/company/press/h22/data/3/0314 (dernière consultation le 26 juillet 2020).

24. Files d'attente continues de véhicules bloquant tout un réseau de rues qui se croisent et stoppant alors complètement la circulation dans toutes les directions.

25. 《Tōkyō-to kitaku kon.nansha taisaku jisshi keikaku » 東京都帰宅困難者対策実施計 画 (Plan de mise en œuvre des mesures pour les personnes qui ne peuvent pas rentrer chez elles à Tokyo), MLIT. 
ont grimpé en flèche dans tout le Sud du Kantō (fig. 02) et la plupart des magasins de vélo sont restés ouverts jusqu'au milieu de la nuit et épuisement total de leur stock ${ }^{26}$. Asahi, un important revendeur de bicyclettes, a déclaré que leurs ventes dans la région ont doublé au cours du mois suivant le tremblement de terre. D'autres département ruraux comme Yamagata ont enregistré une hausse des ventes dans les jours suivant la catastrophe ${ }^{27}$. La faible densité urbaine des zones rurales a néanmoins eu raison de cette envolée fugace, avec des parcours souvent trop longs pour être effectués en bicyclette au quotidien. Si, depuis les années 1990, de plus en plus de navetteurs dans les zones urbaines choisissent de se rendre au travail à vélo pour différentes raisons personnelles, environnementales ou économiques, la catastrophe du 11 mars a rappelé à beaucoup la fiabilité de la bicyclette comme moyen de transport individuel. Elle constituait déjà un objet de valeur pour des millions de personnes dans le Japon d'après-guerre, caractérisé par un système de transport exsangue, des routes en piteux état et des manques chroniques de carburant. Kobayashi Shigeki 小林成基, président du Jitensha katsuyō suishin kenkyūkai 自転車活用推進研究会 (Groupe d'étude pour la promotion de l'utilisation de la bicyclette), a déclaré que le séisme a eu une influence non seulement sur le nombre de cyclistes mais a également modifié de manière significative la culture du vélo au Japon ${ }^{28}$. Quant aux zones touchées par le tsunami, des groupes de bénévoles ainsi que des fabricants se sont mobilisés pour envoyer des bicyclettes afin d'aider les réfugiés. Le constructeur taiwanais GIANT a ainsi envoyé 1000 exemplaires d'un vélo en édition limitée shinsai fukkō shienhin 震災復興支援品 (aide à la reconstruction après le tremblement

26. https://www.shikoku-np.co.jp/national/life_topic/20110413000390 (dernière consultation le 26 juillet 2020).

27. "Shigoto mo seikatsu mo jitensha shifuto ken.nai, chōki-ka suru gasorin fusoku » 仕事も生活も自転車シフト 県内、長期化するガソリン不足 (Changement de style de vie par la bicyclette en raison des pénuries de carburant) : https://www.yamagata-np.jp/feature/ shinsai/kj_2011032400864.php (dernière consultation le 26 juillet 2020).

28. Interview de Kogayashi Shigeki : https://mainichi.jp/articles/20180831/ org/00m/010/045000c (dernière consultation le 26 juillet 2020). 
de terre $)^{29}$. Ces vélos tout-terrain, haut de gamme et robustes, ont été spécialement conçus pour être utilisés par des organisations d'urgence locales et des volontaires sur des routes qui n'étaient plus accessibles aux véhicules motorisés.
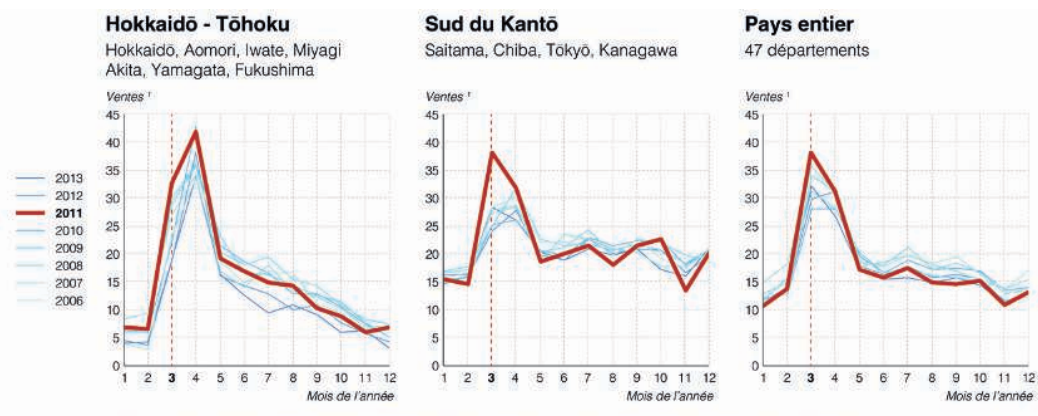

Fig. 02

Ventes de vélos selon le mois de l'année de 2006 à 2013.

Sources : Jitensha sangyō shinkō kyōkai 自転車産業振興協会， Japan Bicycle Promotion Institute30. Réalisation : Martial (2021).

29. Communiqué de presse GIANT: https://web.archive.org/web/20110410060207/ http://www.giant.co.jp/information/2011/04/post-18.php (archive du 5 avril 2011, consultée le 26 juillet 2020).

30. Données du Japan Bicycle Promotion Institute, compilées à partir des statistiques de ventes de 2006 à 2013: http://www.jbpi.or.jp/statistics_list.cgi?cid=10 (dernière consultation le 26 juillet 2020). 


\section{Fig. 03}

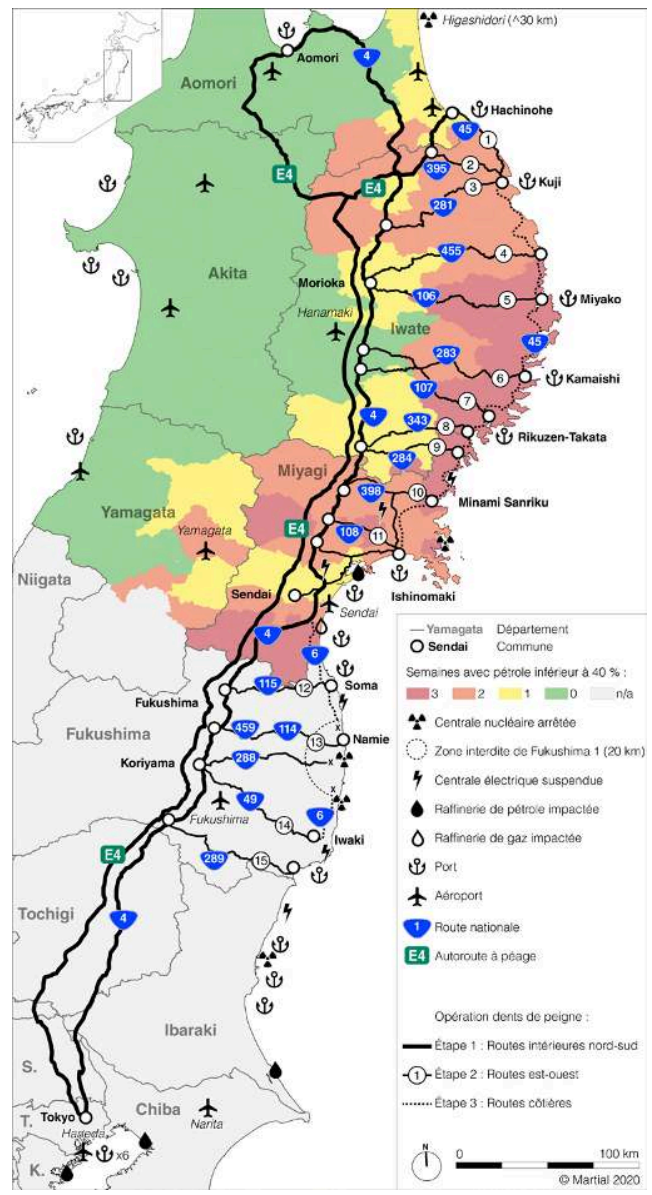

Situation des transports routier, aérien et maritime après le 11 mars 2011.

Sources: Akamatsu et al. 2016, PAJ ${ }^{31}$, MLIT $^{32}$. Réalisation: Martial (2021).

31. Rapport de la PAJ disponible sur le site du Bōsai jōhō 防災情報 (Centre de gestion des catastrophes au Japon): http://www.bousai.go.jp/jishin/syuto/taisaku_wg/10/pdf/ sub2.pdf (dernière consultation le 26 juillet 2020).

32. MLIT, Chronologie de l'opération dents de peigne: http://www.thr.mlit.go.jp/ road/jisinkannrenjouhou_110311/kushinohasakusen.html (dernière consultation le 26 juillet 2020). 


\section{2. Le transport ferroviaire}

La East Japan Railway Company (JR East) et les autres opérateurs ferroviaires ont immédiatement interrompu leurs services après le tremblement de terre. Le personnel des gares, les conducteurs et chefs de train ont évacué les passagers dans toutes les zones où un avertissement de tsunami a été émis, évitant ainsi toute perte humaine parmi les passagers (Hayashi 2012).

Le train à grande vitesse Shinkansen circule sur des voies dédiées, différentes de celles du réseau ferroviaire conventionnel. La ligne Tōhoku Shinkansen opérée par JR East a subi des dommages importants sur environ 1200 sites le long de quelques $500 \mathrm{~km}$ entre les gares d'Ōmiya et de Iwate-Numakunai, notamment l'inclinaison, voire l'effondrement, de poteaux électriques, la rupture de caténaires et des dommages aux piles des viaducs par cisaillement. Seuls 49 jours ont été nécessaires pour la remettre entièrement en service, contre 81 jours pour la restauration de la ligne San.yō Shinkansen à la suite du séisme de 1995 à Kobe, ayant notamment causé l'effondrement de huit ponts. Le tremblement de terre de Chūetsu à Niigata en 2004 avait quant à lui endommagé plusieurs tunnels, nécessitant une mise hors service de la ligne Jōetsu Shinkansen durant plus de deux mois. Ce dernier a par ailleurs occasionné le seul déraillement de Shinkansen recensé à ce jour, ne causant toutefois aucun mort ni blessé. Le Shinkansen et les autres trains à grande vitesse sont en effet conçus pour ne pas se plier lors d'un déraillement, évitant ainsi l'encastrement des voitures, phénomène le plus meurtrier lors d'un accident ferroviaire. La ligne Tōhoku Shinkansen a pu être restaurée rapidement, malgré l'intensité inégalée des secousses sismiques du 11 mars, grâce à trois facteurs essentiels. Le premier a été le système d'alerte précoce aux tremblements de terre mis en place progressivement au cours des dernières décennies avec des sismographes installés sur le littoral du département de Miyagi à partir de 1982, qui ont détecté les ondes sismiques 12 à 15 secondes avant qu'elles n'atteignent les Shinkansen circulant à pleine vitesse. Cette détection précoce a permis d'éviter de lourds dégâts et tout déraillement, en déclenchant un système d'alerte installé en 1998, puis en activant simultanément la fonction de coupure d'alimentation de la ligne installée en 2006, pour enfin activer les freins d'urgence de toutes les voitures en circulation. Le deuxième facteur consiste en de grandes campagnes de nouvelles conceptions antisismiques et de 
rénovations débutées en 1996 à la suite du séisme à Kobe l'année précédente. Malgré l'intensité plus importante du séisme de 2011, les dégâts ont été moindres et aucun n'a été critique; aucune pile renversée comme en 1995 et aucun tunnel effondré comme en 2004. Le troisième facteur essentiel a été l'effort exhaustif de restauration des infrastructures. En effet, si les milliers d'ingénieurs de la Kinkyūsaigai taisaku haken-tai 緊急災害対策派遣 (Technical Emergency Control Force, TEC-FORCE ${ }^{33}$ ) dotés d'installations de communications, d'équipements et de véhicules lourds ont mené jour et nuit une large gamme de travaux de restauration, environ 8500 personnes ont quotidiennement participé à la reconstruction du réseau ferroviaire, comprenant à la fois des employés de JR East, de ses filiales et d'autres entreprises partenaires. Le secteur privé a ainsi démontré son importance dans les opérations d'urgence et de reprise. Cette coopération a pu se concrétiser malgré des approvisionnements en carburant ténus et irréguliers. D'autres opérateurs ferroviaires ont fourni du personnel pour les inspections sur site et les travaux de restauration, ainsi qu'une aide matérielle allant des véhicules d'entretien à l'équipement d'inspection en passant par un soutien en approvisionnement en carburant ${ }^{34}$. Ces différentes collaborations des secteurs publics et privés sont essentielles dans un contexte d'affaiblissement des capacités du secteur privé. Le nombre de sociétés de construction a en effet diminué ces dernières années en raison de la réduction du budget de l'État consacré à la construction d'infrastructures, le Japon ayant atteint une certaine maturation à ce niveau. Le secteur privé peut de moins en moins se permettre d'exclusivement posséder des équipements lourds ou même d'employer de nouveaux ingénieurs et techniciens. Les travaux de restauration se sont toutefois poursuivis sans interruption, permettant la reprise du service de la ligne Tōhoku Shinkansen le 29 avril 2011. Il est à noter que malgré l'absence de dommages dans les régions administrées par les autres compagnies du groupement Japan Railways, ces dernières ont connu des

33. MLIT : https://www.mlit.go.jp/river/bousai/pch-tec/index.html (dernière consultation le 26 juillet 2020).

34. «Yomigaere! Michinoku no tetsudō : Higashi Nihon daishinsai kara no fukkō no kiseki »よみがえれ! みちのくの鉄道: 東日本大震災からの復興の軌跡 (La relance ! Le chemin de fer à Michinoku : reconstruction suite au grand tremblement de terre), MLIT, 2012. 
difficultés dans leur approvisionnement en pièces détachées produites dans les régions touchées, occasionnant des suspensions de service de certains trains opérés par la West Japan Railway Company (JR West) en particulier $^{35}$. Les lignes conventionnelles ont également été sévèrement touchées, avec 1730 sites endommagés uniquement par le tremblement de terre et 4400 par le séisme et le tsunami (fig. 04). Ils ont été répertoriés le long de trente-six segments ferroviaires avec, de nouveau, l'inclinaison voire l'effondrement de poteaux électriques, des irrégularités de voies avec notamment l'affaissement du ballast ou encore des destructions de quais de gare. Sur les sept segments de lignes conventionnelles endommagés par le tsunami, quelque vingt-trois bâtiments de gare, $60 \mathrm{~km}$ de voies et des piliers de pont sur cent-un sites ont été emportés ou enfouis par les flots. Les répliques intermittentes à partir du 7 avril ont exacerbé les dégâts et entraîné de nouvelles interruptions de service sur les lignes alors déjà remises en état. Celles-ci ont néanmoins pu redémarrer en quelques jours.

Un état des lieux synthétique est présenté du nord au sud de la côte Pacifique pour plus de clarté. La ligne la plus septentrionale ayant connu des avaries a été la ligne Ōminato opérée par JR East. Les lignes Kitariasu (Rias-Nord) et Minamiriasu (Rias-Sud), gérées par le groupe Sanriku tetsudō 三陸鉄道 (Sanriku Railway) du daisan sekutā 第三セクター (troisième secteur ${ }^{36}$ ) et séparées avant le tremblement de 2011 par la ligne JR Yamada entre Miyako et Kamaishi, ont été parmi les plus sévèrement touchées (Makoto et al. 2011) avec des dommages importants causés aux bâtiments de gare et aux ponts sur plus de 300 endroits. Le tsunami a emporté près de $6 \mathrm{~km}$ de voies et le service n'a repris qu'en avril 2014 avec de nouvelles voitures diesel ${ }^{37}$. Mais les deux lignes sont néanmoins restées séparées par le segment endommagé de l'ancienne ligne Yamada, originellement composée de deux sections; la première dans les terres, reliant Morioka à Miyako, la seconde longeant la Sanriku kaigan (côte Sanriku) de Miyako à Kamaishi. Si la première n’a été suspendue qu'un

35. JR-West annual report 2011.

36. Définit une multiplicité d'acteurs à cheval entre secteurs public et privé; ici les diverses compagnies ferroviaires privées nipponnes, à but lucratif, sont soumises à de nombreuses régulations pour assurer un service public.

37. Notamment grâce au soutien du Koweït, fournissant carburant et locomotives : https://www.sanrikutetsudou.com/?p=993 (dernière consultation le 26 juillet 2020). 
mois, la seconde a été profondément impactée, avec plus de $21 \mathrm{~km}$ de voies inondées, quatre des treize gares, un dixième des voies, six ponts et dix remblais détruits (Kazama \& Noda 2012). Japan Railways proposa en 2012 que la section endommagée soit mise au rebut afin que son emprise soit utilisée comme voie de transport rapide pour un Bus Rapid Transit $(B R T)^{38}$. Cette décision a été abrogée en 2015 et la section a été reconstruite avec pour objectif de la transférer ultérieurement à Sanriku Railway. Il a au total fallu huit ans pour rouvrir cette section en 2019, permettant de relier les deux lignes Rias-Nord et Rias-Sud et ainsi créer la ligne Riasu (Rias). Il est désormais possible de relier directement les gares de Kuji et Sakari, distantes de 163 km, en un peu plus de quatre heures, la ligne ne proposant que des trains omnibus à vitesse modeste. La ligne a été de nouveau suspendue après le passage du super typhon Hagibis en 2019.

Les mécanismes de soutien et de réhabilitation ont été établis au cas par cas depuis plus d'un demi-siècle sans perspective globale ${ }^{39}$ et ne couvrent généralement pas les infrastructures appartenant au secteur privé. Le fait que Sanriku Railway soit une propriété conjointe des gouvernements locaux et du secteur privé explique ses difficultés. La reconstruction de ses lignes ferroviaires a nécessité la somme considérable de 18 milliards de yens, représentant une énorme charge pour la compagnie qui souffrait déjà de difficultés économiques dans une zone rurale en dépeuplement continu. Le gouvernement national a, dans un premier temps, proposé de prendre en charge $25 \%$ des coûts de reconstruction dans le cadre des lois existantes, laissant donc une part majeure aux gouvernements locaux pour le soutien du groupe privé. Cela réduit fortement les marges de manœuvre des autorités locales et ne permet pas d'améliorer les usages d'une infrastructure endommagée. Cette rigidité peut en outre décourager l'investissement régulier dans la gestion des risques au quotidien en

38. Souvent traduit en français par Bus à haut niveau de service (BHNS), se définit comme une ligne de bus avec une priorité garantie, une vitesse et une fréquence élevées, une plage horaire ample, accessible aux personnes à mobilité réduite et une identification forte.

39. Kōeki shadanhōjin doboku gakkai 公益社団法人土木学会 (JSCE, Japan Society of Civil Engineers) : http://www.jsce.or.jp/committee/editorial/files/no25_morichi.pdf (dernière consultation le 26 juillet 2020) 
préparation de la prochaine catastrophe et peut même inciter les gouvernements locaux à "attendre les désastres " pour mener à bien des projets d'envergure, le gouvernement central étant alors plus prompt à accorder des subventions plus élevées aux projets de reconstruction que d'entretien ou d'anticipation ${ }^{40}$.

Le terminus Sakari de la ligne Rias-sud offre un changement pour la ligne Ōfunato, dont la partie côtière a été lourdement affectée. Si le service a vite repris sur la section ouest de $62 \mathrm{~km}$ entre Ichinoseki et Kesennuma, la section de 43,7 km entre Kesennuma et Sakari est restée fermée après que JR East a officiellement décidé de sa fermeture, afin que son emprise soit utilisée pour la création de la ligne Kesennumasen ōfunatosen bīārutī (BRT entre Kessennuma et Ōfunato). De la même manière, la ligne Kesennuma a été partiellement fermée et remplacée par un BRT à la suite des coûts prohibitifs de restauration de la voie ferrée et des bâtiments de gare détruits entre autres à Minami-Kesennuma et Shizugawa. Aujourd'hui, sur l'ancienne ligne de $72,8 \mathrm{~km}$, seul le tronçon de 17,5 km entre Maeyachi et Yanaizu demeure exploité par voie ferrée. L'ancien terminus Kesennuma n'est désormais accessible qu'avec la ligne Ōfunato. Les lignes Ōfunato et Kesennuma représentent ainsi les seules non entièrement reconstruites ou déplacées ${ }^{41}$, avec un total de $99 \mathrm{~km}$ de voies ferrées pour la plupart requalifiées en routes bitumées ou en voies réservées aux BRT.

Plus au sud, la ligne côtière Jōban a été l'une des plus touchées. La plupart des tronçons ont rouvert dans les jours, semaines ou mois qui ont suivi, mais les dégâts les plus importants occasionnés par le tsunami ont été répertoriés entre les stations Hama-Yoshida et Komagamine, cette dernière se situant à moins de $50 \mathrm{~km}$ au nord de la centrale nucléaire de Fukushima Daiichi. Ces dommages ont induit une relocalisation partielle de la ligne plus à l'intérieur des terres, dans le cadre du plan de restauration de la ville (Fujimoto et al. 2018). La ligne Jōban passe

40. “Keizai shakai sōgōkenkyūsho » 経済社会総合研究所 (ESRI : Economic and Social Research Institute) : http://www.esri.go.jp/jp/prj/hou/hou044/hou44-6-1.pdf (dernière consultation le 26 juillet 2020)

41. https://www.tetsudo.com/special/disaster2011 (dernière consultation le 26 juillet 2020). 
également à proximité immédiate de la centrale nucléaire, avec les gares de Futaba et Ōno à moins de $10 \mathrm{~km}$, et un tronçon s'approchant à moins de $5 \mathrm{~km}$. La reprise des opérations du dernier tronçon inopérant de 20,8 km allant des gares de Namie à Tatsuta a été décidée en 2015, dans l'objectif de rouvrir toute la ligne à l'horizon 2019. Après la réouverture de la station Tomioka le 21 octobre 2017, la ligne a été totalement remise en service le 14 mars $2020^{42}$, représentant ainsi la fin des travaux de reconstruction ferroviaire dans l'Archipel à la suite du tremblement de terre (voir tableau 01). Les conditions de reconstruction de ce tronçon, extrêmement complexes en raison de la contamination radioactive, de l'existence prolongée et de la révision continue des zones d'évacuation, du grand nombre d'évacués et de la nécessité du stockage et de l'élimination des sols contaminés, ont eu des incidences profondes sur l'infrastructure locale des transports en commun, la disponibilité en matériel de construction et de main-d'œuvre.

42. https://mainichi.jp/articles/20200314/k00/00m/040/003000c (dernière consultation le 26 juillet 2020). 


\section{TABLEAU 01}

Détail des voies ferroviaires (passagers) impactées par le séisme du 11 mars 2011.

\begin{tabular}{|c|c|c|c|c|c|}
\hline & Ligne & Date de reprise totale (1) & Terminus & (5) & (6) \\
\hline & Japan Railways & & & & \\
\hline & 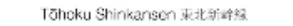 & 29 amil 2011 & Tckyo- Hachirone & 593,1 & 49 \\
\hline 1 & Ōminato $大$ w & 17 mars 2011 & Nahej - Ominata & 58.4 & 6 \\
\hline 2 & Tsugaru $=$ = & 15 mars 2011 (10 awril 2011) & Acmori - Mimmaya & 55,8 & 7 \\
\hline 3 & 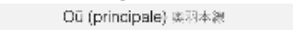 & 31 mars 2011 (11 avril 2011$)$ & Fukushima - Àcmeri & 486,3 & 24 \\
\hline 4 & Geno 不堆沼 & 19 mars 2011 (10 avril 2011) & H. Noshiro - Kawabe & 147,2 & 11 \\
\hline 5 & Hechinche $\triangle A F$ & $17 \mathrm{mars} 2012$ & Hachinche - Kuji & 64,9 & 372 \\
\hline 6 & Hanawa 花棌析 & 19 mars 2011 (11 avril 2011) & Kčma-Odate & 106,9 & 12 \\
\hline 7 & Oga $\mp$ es & 15 mars 2011 & Owake - Oga & 26,6 & 4 \\
\hline 8 & Yamada 山口及 & 23 mars $2019(2)$ & Morika - Myako & 102,1 & 2934 \\
\hline 9 & Tazawake E以: & 18 mars 2011 (9 avril 2011) & Mcrioka - Ōmegari & 75,6 & 10 \\
\hline 10 & Kamaishi 羊不流 & 6 avril 2011 (12 avril 2011) & Hanamaki - Kamaishi & 90,2 & 31 \\
\hline 11 & 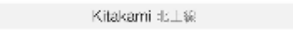 & 20 mars 2011 (11 avril 2011) & Kitakami - Yokote & 61,6 & 13 \\
\hline 12 & 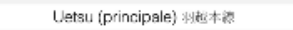 & 14 mars 2011 (9 avil 2011) & Nitsu - Akita & 274,4 & 5 \\
\hline 13 & Olunato tfux & 1er awril 2011 (18 avril 2011) (3) & IChincseki - Sakari & 105,7 & . \\
\hline 14 & Kesennuma 婪仙洋滕 & 29 avril 2011 (4) & Maeyachi - Kesennuma & 72,8 & - \\
\hline 15 & Ishinamaki 不蕉綿 & 21 mars 2015 & Keguta - Onagava & 44,9 & 1471 \\
\hline 16 & Rilkuu ouest f5: & $1^{\prime \prime \prime}$ avil 2011 (9 avril 2011) & Shinjo-Amarume & 43,0 & 23 \\
\hline 17 & 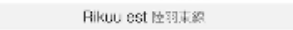 & 3 avril 2011 (16 asril 2011) & Kogala-Shir $\delta$ & 94,4 & 32 \\
\hline 18 & Sensekj 山石祀 & 30 mai 2015 & Aoba-dōri - Ishincmaki & 50,8 & 1541 \\
\hline 19 & Senzan $1:$ in & 23 amila11 2011 & Sendai - Uzen Chilose & 58,0 & 12 \\
\hline 20 & Aterazawa ¿.下䋆 & 28 mars 2011 (9 avril 2011) & K.-Yarnagata - Aterazawa & 24,3 & 19 \\
\hline 21 & Yonesaka + & 20 mars 2011 & Yonezawa - Sakamachi & 90,7 & $\mathrm{~g}$ \\
\hline 22 & Têroku (principale) stu位 & 21 avril 2011 & Tckyo - Moricka & 575,7 & 41 \\
\hline 23 & Joban $x$ sets & 14 mars $20 \times 0$ & Nopori - Isanuma & 351,0 & 3291 \\
\hline 24 & 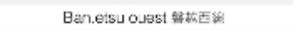 & 26 mars 2011 & Kōriłama - Niitau & 175,6 & 15 \\
\hline 25 & 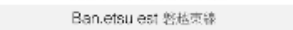 & 15 sanil 2011 & lwaki-Kơryama & 85,6 & 35 \\
\hline 26 & Sugun $*$ age & 11 avril 2011 & Mito - Asaka-Nagamori & 147,0 & 31 \\
\hline 27 & Tadami hra & 14 swil 2011 & Aizu-Wakamats - Koide & 135,2 & 34 \\
\hline & Autres & & & & \\
\hline A & 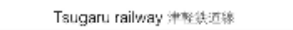 & 13 mars 2011 & Tsugaru-G. - Tsugaru-N. & 20,7 & 2 \\
\hline B & 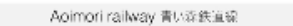 & 17 mars $2011(10$ awril 2011$)$ & Matoki-Aamori & 121,9 & 9 \\
\hline c & 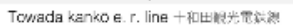 & 13 mars 2011 & Misawa - Towadashi & 14.7 & 2 \\
\hline $\mathrm{D}$ & 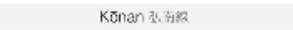 & 14 mars 2011 & Hircsaki - Kuroisni & 16,8 & 3 \\
\hline E & Owari tign & 14 mars 2011 & Cuan - Chūn̄-Hircsaki & 13.9 & 3 \\
\hline $\mathrm{F}$ & Iwate Galaxy railyay uns & 17 mars 2011 (10 awril 2011) & Morioka-Moloki & 82,0 & 9 \\
\hline G & Akita Nairiku 秋世内陡絲 & 22 mars 2011 & Takanosu - Kakunodate & 94,2 & 11 \\
\hline H & 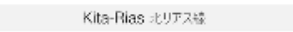 & 23 mars $20+9(2)$ & Kuji - Miyako & 71,0 & 2934 \\
\hline 1 & Minami-Rias muy ג仿 & 23 mars $2019(2)$ & Kamaishi-Sekar & 36,6 & 2934 \\
\hline J & Conkai Sanroku s: & 13 mars 2011 & Ugo-Hen $\delta$ - Yashima & 23,0 & 2 \\
\hline $\mathrm{k}$ & 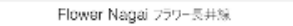 & 20 mars 2011 (9 avril 2011) & Akay - Arato & 30,5 & 11 \\
\hline L & Nanboxu (merto de Senda) 南北投 & 23 gพmil 2011 & Izumi-Choo-Tomizawa & 14,8 & 49 \\
\hline M & 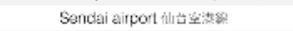 & $1^{\text {wit }}$ octoure 2011 & Na:ori - AǴroport & 7,1 & 204 \\
\hline $\mathrm{N}$ & | Zaka of the? & 13 mars 2011 & Fusushima - lizaka onsen & 9,2 & 2 \\
\hline o & 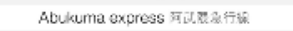 & 16 mai 2011 & Fukushima - Tsukincki & 54,9 & 66 \\
\hline $\mathrm{P}$ & Minatn a & 23 . jillet 2011 & Katsuta-Aijigaura & 14.3 & 134 \\
\hline o & Oarai Kashima $x$ 狧f & 12 jullbt 2011 & Milo - Kashima-Jingū & 53,0 & 123 \\
\hline $\mathrm{R}$ & Kashima itsin & 16 samil 2011 & Katori - Kssh-ima-lingü & 17,4 & 36 \\
\hline & & 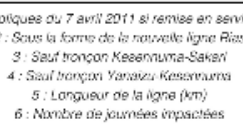 & ele evant & & \\
\hline
\end{tabular}

Source : «Yomigaere! Michinoku no tetsudō : Higashi Nihon daishinsai kara no fukkō no kiseki » よみがえれ! みちのくの鉄道: 東日本大震災からの復興の軌跡 (La relance! Le chemin de fer à Michinoku : reconstruction suite au grand tremblement de terre).

Réalisation : Martial (2021). 


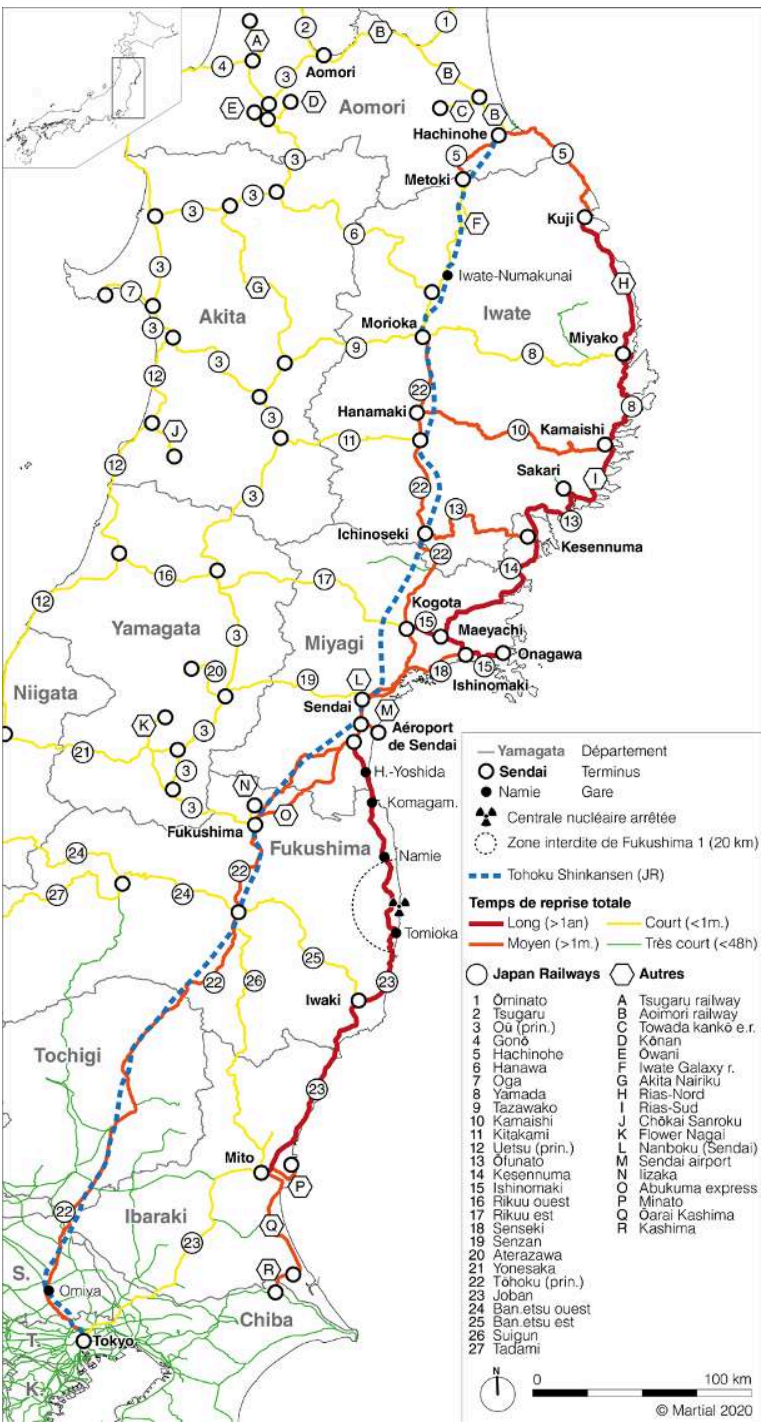

Fig. 04

Voies ferroviaires (passagers) impactées par le séisme du 11 mars 2011.

Source : «Yomigaere! Michinoku no tetsudō : Higashi Nihon daishinsai kara no fukkō no kiseki »よみがえれ!みちのくの鉄道: 東日本大震災からの復興の軌跡 (La relance! Le chemin de fer à Michinoku : reconstruction suite au grand tremblement de terre). Réalisation : Martial (2021). 
L'ampleur de la catastrophe sismique, les bouleversements démographiques des zones touchées par la catastrophe nucléaire et les incertitudes découlant de la contamination radioactive continuent en effet de rendre difficiles l'exploitation et la planification des transports locaux. Si la plupart des transports locaux étaient paralysés à la suite de la catastrophe, d'autres ont pu fournir des moyens essentiels lors de l'évacuation d'urgence dans un premier temps, puis dans la période de transition, afin de répondre à la demande changeante induite par l'actualisation de la zone d'évacuation. De nouvelles lignes de bus, des BRT mais aussi des navettes à la demande, ont ainsi été mises en place. Les BRT se prêtent souvent mieux au transport de passagers dans des zones rurales de faible densité, mais ne desservent pas de destinations précises comme les hôpitaux ou les écoles, au contraire des bus municipaux, plus lents mais aux arrêts plus nombreux et moins éloignés. Ainsi, une ONG a pu coordonner l'accès aux services publics, supermarchés et autres destinations du quotidien grâce à différents services de transport bénévoles, contribuant à la mobilité des évacués, notamment à Ōfunato. Le faible approvisionnement en produits pétroliers a eu moins d'effet sur les taxis et bus de la région que sur les particuliers : les taxis fonctionnent majoritairement au gaz de pétrole liquéfié et les bus ont pu compter sur la Nihon basu kyōkai 日本バス協会 (Association nationale des bus) pour acheminer du carburant en urgence dans la région depuis leurs réserves disséminées sur tout le territoire. Ces nouveaux transports étant néanmoins grandement dépendants de financements débloqués en 2011 par le MLIT pour une période de dix ans jusquà fin 2020, leur statut sur le long terme semble donc précaire. Une partie de ce budget a, par exemple, été allouée afin de relier les villages de réfugiés à la population en continuelle baisse, mais les compagnies de bus semblent tarder à adapter leurs modèles économiques à la fin des allocations du gouvernement. Il faut en outre noter que la région du Tōhoku connaît un phénomène mai kāマイカー (culture de l'automobile, de l'anglais my car) important, les réseaux de transports publics se révélant inadaptés aux dynamiques d'étalement urbain. Une grande majorité de personnes ont de ce fait racheté un véhicule privé dès que possible et elles n'ont pas souhaité ou guère pu changer leurs habitudes de mobilité (fig. 05). Le dynamisme et la densité des transports de Sendai demeurent un contre-exemple pour la région : la plupart des lignes de trains fermées suite à la catastrophe avaient comme la plupart des lignes de province une santé économique chancelante. Les 
transports ferroviaires sont en effet des investissements nécessitant une grande densité urbaine pour espérer atteindre la rentabilité. Les BRT mis en place proposent un service de haute qualité pour un investissement bien moindre, mais ils ne forment qu'une solution parmi d'autres à l'heure d'une émergence des smart grids et smart communities, qui proposent à terme différentes solutions de transports à la demande.

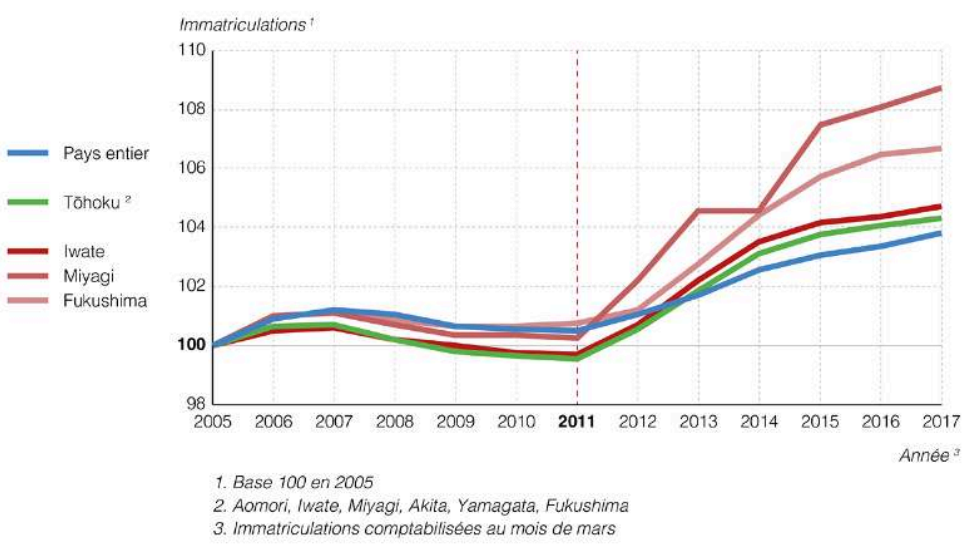

Fig. 05

Évolution du nombre de véhicules motorisés immatriculés au Japon de 2005 à 2017.

Source : Ji ken-kyō 自検協, AIRIA ${ }^{43}$.

Réalisation: Martial (2021).

Le nombre de véhicules motorisés en circulation n'a pas connu de baisse notable en 2011 malgré les 400000 véhicules endommagés ou détruits, indiquant un rachat quasi systématique dans l'année. Si la hausse du nombre d'immatriculations est relativement continue au niveau national, il faut noter une inflexion en 2011 dans le Tōhoku, en particulier dans les

43. Abréviation de Jidōsha kensa tōroku jōhō kyōkai 自動車検査登録情報協会 (Automobile Inspection \& Registration Information Association). Statistiques d'immatriculation : https://www.airia.or.jp/publish/statistics/number.html (dernière consultation le 26 juillet 2020) 
départements de Miyagi et de Fukushima, qui ont le plus souffert de la baisse sensible du niveau de service des transports en commun.

\section{3. Le transport aérien}

La situation critique des transports routiers et ferroviaires transforma le transport aérien, dès lors considéré comme la solution la plus praticable et efficace pour accéder aux régions touchées pendant au moins les vingt jours suivant la catastrophe. Quelques instants après la première secousse sismique, l'aéroport de Sendai, situé à quelques centaines de mètres de la côte, ne disposant pas de digues suffisantes et de brise-lames, a été frappé de plein fouet par le tsunami. Les deux pistes, l'aire de stationnement et les voies de circulation ont été submergées par plusieurs mètres d'eau et le rez-de-chaussée du terminal a été presque entièrement inondé. Toutefois, les quelque 1200 passagers et membres du personnel ayant eu le temps de se réfugier sur les niveaux supérieurs, aucun mort ou disparu n'a été déploré dans l'enceinte de l'aéroport. Les groupes électrogènes de secours ainsi que le radar au sol du contrôle aérien ont été détruits. Dans des conditions normales, l'aéroport de Sendai constitue une plaque tournante du trafic aérien du Tōhoku, les autres aéroports de la région ne desservant généralement qu'un nombre limité de passagers et de marchandises. Sa remise en service constituait donc une priorité absolue pour les autorités.

Les Forces armées des États-Unis au Japon ont fourni hommes et moyens dans le cadre de l'opération d'aide humanitaire cruciale Tomodachi sakusen トモダチ作戦 (opération Tomodachi). Elle s'est déroulée du 12 mars au 4 mai 2011, a impliqué 24000 militaires américains, 189 avions, 24 navires de guerre et a coûté 90 millions de dollars. La majorité des bases militaires américaines de l'Archipel ont été mobilisées et ont mené des opérations conjointes avec les Forces japonaises d'autodéfense (Wilson 2012). L'armée américaine ayant constamment besoin d'une grande quantité de carburant pour fonctionner, elle a pu s'appuyer sur ses réserves conséquentes. Tandis que les pompiers et volontaires s'affairaient généralement à l'assistance des individus, la mobilisation des forces militaires a permis non seulement l'évacuation, la construction d'abris d'urgence, de sanitaires et de douches, mais aussi la réouverture de l'aéroport de Sendai dès le 15 mars pour les vols humanitaires, qui s'est alors transformé en plaque tournante du transport de fournitures d'urgence, malgré les quelques 2000 voitures endommagées 
et $370000 \mathrm{~m}^{3}$ de débris charriés sur son emprise. Les hélicoptères sont arrivés dès le quatrième jour après la catastrophe et les avions de transport des forces armées américaines dès le cinquième, après l'installation à même la piste d'atterrissage du matériel nécessaire à un contrôle aérien temporaire. L'aéroport a rouvert le 13 avril pour les vols commerciaux, soit seulement 32 jours après la catastrophe, et a retrouvé un fonctionnement normal le 25 septembre 2011.

Des alternatives d'urgence pour le transport aérien civil ont néanmoins été nécessaires jusqu'à la restauration complète de l'aéroport de Sendai. Les autorités ont choisi d'exploiter la capacité excédentaire de certains aéroports régionaux du Tōhoku, en maintenant ainsi un niveau de service sensiblement identique après la catastrophe, solution qui consistait à dérouter les vols de l'aéroport inopérant de Sendai vers ceux de Hanamaki, de Fukushima et de Yamagata, entrainant une augmentation conséquente de leur nombre de passagers. L'aéroport de Yamagata, grâce à sa proximité avec la ville de Sendai, sa faible contamination radioactive et ses dégâts mineurs, a multiplié par quinze son nombre de passagers durant les vingt jours après la catastrophe. Il passe ainsi de 20 à 60 appareils par jour, tandis que Hanamaki et Fukushima passent respectivement de 20 à 100 et de 25 à 85 (Aratani et al. 2013). Malgré la rapidité et le degré de flexibilité surprenants de ces changements, certaines opérations ont atteint des seuils critiques, comme la gestion du stationnement des avions et du grand nombre d'hélicoptères, ainsi que l'approvisionnement en carburant. Les responsables de la gestion de la situation d'urgence ont tenté d'adapter au mieux la capacité de transport de l'aéroport de Yamagata face à une demande croissante, mais il n'a malgré tout pas été possible de garantir un vol à tous les passagers arrivant au terminal durant les premiers jours. La remarquable hausse de la demande de transport terrestre vers l'aéroport s'est répercutée sur les transports publics régionaux : ajouté à l'indisponibilité des chemins de fer, le nouveau rôle conféré à l'aéroport de Yamagata a pesé sur le système de transport terrestre du Tōhoku.

Les aéroports de Haneda et de Narita desservant la capitale, de loin les plus fréquentés de l'Archipel, ont suspendu leurs activités quelques minutes après la première secousse sismique, malgré l'absence de dégâts majeurs sur les pistes ou les terminaux. Ainsi, sur les 500 et 250 avions quotidiens 
qu'accueillent respectivement les deux aéroports, 86 ont été déroutés ${ }^{44}$. Véritable carrefour dans l'assistance par voie aérienne durant l'opération Tomodachi ${ }^{45}$ et l'évacuation de familles américaines ${ }^{46}$, la base aérienne américaine de Yokota à proximité de Tokyo a également admis 11 appareils américains civils. Au total, 8500 passagers ont rencontré des difficultés pour rentrer chez eux.

À l'exception de celui de Sendai, les aéroports du pays ont pu rouvrir dès le 14 mars. Néanmoins, en raison des craintes concernant l'accident nucléaire, plusieurs compagnies aériennes ont suspendu leur desserte de la capitale à partir du 15 mars. Si chaque aéroport a régulièrement mis à jour les mesures de résistance aux tremblements de terre, la catastrophe de 2011 a illustré l'insuffisance des mesures anti-tsunami, démontré l'importance des aéroports lors d'une catastrophe et aidé à tirer plusieurs enseignements (Hanaoka et al. 2013). Il faut tout d'abord améliorer et simplifier la communication et la coopération entre les différentes organisations aéroportuaires lors d'une situation d'urgence afin d'éviter une trop grande confusion. Le tremblement de terre d'Iwate-Miyagi Nairiku en 2008 a été l'opportunité pour le personnel de l'aéroport de Hanamaki de travailler sur ces questions durant trois ans jusqu'à la veille de la catastrophe, leur permettant de partager rapidement leurs retours d'expériences à travers l'Archipel. D'autres ont entrepris des efforts similaires sur le contrôle du trafic aérien, l'attribution des places de stationnement et le ravitaillement. Le Japan Civil Aviation Bureau (JCAB) a alors pu proposer des formations continues et prendre en charge le contrôle aérien; le gouvernement local devient administrateur de l'aéroport; les exploitants aériens et les gestionnaires des installations aéroportuaires peuvent alors gérer une série de tâches d'exploitation en vol même en cas d'urgence. En outre, la diversification des sources de carburant des aéronefs et la sécurisation des routes d'approvisionnement reposent sur une coordination entre les fournisseurs, les distributeurs de carburant et l'administration des aéroports.

44. https://www.jiji.com/jc/graphics?p=ve_soc_jishin-higashinihon20120401j-01-w470 (dernière consultation le 26 juillet 2020).

45. https://www.stripes.com/news/yokota-transforms-into-hub-of-u-s-military-s-earthquake-relief-operations-1.139135 (dernière consultation le 26 juillet 2020).

46. https://www.stripes.com/news/pacific/japan/military-wraps-up-first-round-of-departures-from-japan-1.138869 (dernière consultation le 26 juillet 2020). 
La coopération entre les exploitants d'aéronefs est cruciale pour assurer une rapidité de décision, primordiale pendant la période initiale de la catastrophe. Les aéronefs gouvernementaux, ceux des Forces japonaises d'autodéfense et des garde-côtes japonais, ceux de l'armée américaine, les hélicoptères d'intervention médicale ou des médias, sans oublier les avions commerciaux assurant le transport d'urgence, ont tous joué un rôle important. Naturellement, chaque organisation impliquée a géré la situation en transmettant ses informations à son siège respectif. Il a été difficile pour le quartier général d'intervention de donner des instructions spécifiques et détaillées au département de l'aviation, une organisation administrative générale étant rarement impliquée dans ces activités. Chaque organisation, ne connaissant que partiellement les actions des autres, a eu du mal à se coordonner. Le caractère immédiat d'une catastrophe entraîne inévitablement des actions entreprises rapidement et indépendamment par chaque organisme, mais un mécanisme pour partager un minimum d'informations utiles comme des statuts d'activités a probablement fait défaut. La mise en place d'une équipe opérationnelle aéronautique dans les phases de transition et de reprise au sein du quartier général d'intervention aurait permis une gestion de crise plus efficace (avec un système doté d'une chaîne de commandement claire permettant le partage des informations, l'implémentation de mesures et d'instructions entre les organisations). La communication entre les aéronefs lors de la catastrophe a joué un rôle essentiel dans le lancement simultané de nombreux vols par plusieurs organisations vers une zone spécifique. Bien que de nombreuses règles existent en temps normal pour assurer la sécurité des vols, la sécurisation d'une méthode de communication commune entre les aéronefs et la station au sol a été précieuse pour recueillir des informations et mener les activités de sauvetage en toute sécurité.

La catastrophe a rappelé la nécessité de transformer les aéroports en bases de gestion de crise et de ravitaillement. La nature du transport aérien, dénué de réseau physique continu et connecté aux destinations nationales et internationales, lui confère une résilience supérieure aux réseaux routiers et ferroviaires. Les marges importantes sur les taux d'exploitation des installations aéroportuaires ont démontré l'importance des surplus de capacité et de certaines redondances pour encaisser une partie de la sévérité du choc d'une catastrophe. La gestion en 2011 a toutefois suggéré une utilisation polyvalente des installations aéroportuaires pour des situations de crise 
ponctuelles en assurant par exemple un espace suffisant de stationnement d'avions, un stockage provisoire supplémentaire de carburant, d'éclairage temporaire pour le travail de nuit ou encore de magasins de triage et de stockage pour les fournitures de secours.

\section{4. Le transport maritime}

Le Japon, nation insulaire, peut compter sur l'étendue de sa zone maritime, son littoral de $34000 \mathrm{~km}$ et son industrie majoritairement implantée sur les zones côtières, à proximité de nombreuses installations portuaires. Les quelque 1100 ports de commerce et 3000 ports de pêche représentent $99 \%$ du tonnage des marchandises transportées dans le pays ${ }^{47}$. Or, avec des vagues pouvant atteindre $9,5 \mathrm{~m}$ comme dans le port d'Ōfunato, le tsunami a dévasté un nombre important de ces installations tout le long de la côte Pacifique. Le port de Sendai a été frappé par le tsunami quelques minutes après la première secousse sismique, ceux de Minamisanriku et Rikuzentaka ont été submergés. Le tsunami a endommagé voire détruit un bon nombre de digues et brise-lames, dont le plus long du monde inauguré en 2009 dans la baie de Kamaishi. La digue de Miyako, longue de 2,5 km, a quant à elle été détériorée à plusieurs endroits. Ces protections ont toutefois démontré leur efficacité pour retarder et abaisser la hauteur de la vague dans certaines zones. D’Aomori à Ibaraki, de nombreux conteneurs ont été éparpillés sur les quais ou le rivage. Additionnés aux différents véhicules, navires, bâtiments et bois charriés, ils sont devenus des obstacles majeurs pour un passage sécurisé dans les différents canaux et zones d'ancrage (Kumagai 2013). Les secours par transport maritime sont donc restés limités le temps de dégager les postes d'amarrage permettant le déchargement. Après la levée de l'alerte au tsunami dans la soirée du 13 mars, le dégagement des différents canaux et installations portuaires a pu commencer dès le lendemain matin. La priorité des travaux de nettoyage a été accordée aux ports de Miyako, Kamaishi et Sendai-Shiogama, le plus grand port du Tōhoku, dans le but prioritaire de transporter des fournitures d'urgence. Le déblaiement a ensuite été étendu vers d'autres grands ports. Le premier

47. Technical standards and commentaries for port and harbors facilities in Japan, MLIT. 
navire transportant des produits de première nécessité a ainsi pu entrer dans le port de Kamaishi dès le 16 mars. Le premier pétrolier a pu s'amarrer dans le port de Sendai-Shiogama le 21 mars, soulageant ainsi la pénurie de carburant dans la région. Le 23 mars, dix ports ont vu leurs passages respectifs sécurisés, permettant un acheminement plus conséquent des secours par voie maritime. Les effets économiques de la destruction des ports ont été sensibles dans toute la région du Tōhoku, surtout dans le département de Miyagi. Son complexe portuaire de Sendai-Shiogama, qui représente environ $40 \%$ du commerce international de la région, se compose de quatre ports : Ishinomaki, Shiogama, Matsushima et Sendai. Si Sendai-Shiogama a rouvert partiellement dès le 16 avril, le volume de containers échangés a diminué de plus de moitié en 2011 et ce n'est qu'en 2015 qu'il a recouvré son niveau de $2010^{48}$. Plus de 300 ports de pêche et près de 20000 de leurs bateaux ont été endommagés voire détruits ${ }^{49}$. Seuls 115 ont pu reprendre toutes leurs activités dans les deux années suivant la catastrophe ${ }^{50}$; fin 2019, 35 d'entre eux avaient toujours une opérabilité limitée ${ }^{51}$.

L'expérience des ports de la côte Pacifique lors de la catastrophe de 2011 offre de multiples enseignements pour les ports du monde entier. L'ampleur des dégâts a non seulement rappelé la vulnérabilité d'une économie, même technologiquement avancée, mais aussi que d'importantes pertes de trafic à long terme peuvent persister, malgré la restauration des infrastructures endommagées. Cela pose des leçons à tirer pour des installations portuaires non seulement confrontées à la menace des catastrophes naturelles, mais aussi d'événements soudains d'origine humaine comme les guerres, les embargos ou encore les grèves générales. Sendai-Shiogama a démontré que l'atténuation avant les catastrophes ou les actions préventives constitue la meilleure option pour faire face aux risques sismiques, grâce au renforcement des sols par densification ou compactage, la conception sismique des structures portuaires, la modernisation des installations intermodales

48. http://www.pa.thr.mlit.go.jp/kakyoin/effort/butsuryu/pdf/dai13kaihonbukai_ shiryou3-1.pdf.

49. Voir note 10 .

50. Fukkōchō 復興庁 (Reconstruction Agency): https://www.reconstruction. go.jp/20130816_FukkoShihyo.pdf (dernière consultation le 26 juillet 2020).

51. Fukkōchō :

https://www.reconstruction.go.jp/topics/main-cat1/subcat1-2/20191122_FukkoShihyo.pdf (dernière consultation le 26 juillet 2020). 
comme les ponts ou bien sûr la mise en place de digues et brise-lames. Face à l'ampleur du tsunami de 2011, aucune de ces mesures n'a suffi. Ces pertes durables sont intrinsèques aux conditions de grande concurrence portuaire, nationales comme internationales. Cette perspective sur le long terme présente une leçon importante pour les planificateurs de catastrophes, surtout concernant des installations aussi capitalistiques que les ports. La perturbation causée par des dommages majeurs soudains peut entraîner à court terme une chute des revenus portuaires et à long terme un affaiblissement de position concurrentielle. Les modèles classiques des conséquences économiques des catastrophes naturelles supposent en effet un rétablissement complet une fois les dommages physiques entièrement réparés. Le 11 mars 2011 a pourtant montré leur obsolescence et la nécessité de nouveaux modèles pour faire face aux potentielles retombées économiques qui perdureraient au-delà de la période de reconstruction physique.

\section{5. Les télécommunications}

Les premières évaluations des dommages et sauvetages exigent des outils de communication performants, rendant les réseaux de télécommunications cruciaux lors d'une catastrophe. Les services dans les zones touchées sont soumis à des demandes bien supérieures à la normale, plaçant les gestionnaires dans une situation de fonctionnement d'urgence même en l'absence de dommages physiques sur le réseau ${ }^{52}$. La catastrophe du 11 mars a permis d'ouvrir un vaste domaine d'améliorations des performances lors d'une situation critique. Un renforcement sismique des bâtiments et du matériel informatique a par le passé permis de réduire les dommages, mais les raccordements n'ont pour la plupart pas été mis à niveau. Ces derniers ont été à nouveau endommagés par les contraintes géologiques considérables lors d'un séisme, tels que la liquéfaction, la déformation du sol ou encore les glissements de terrain.

52. Les principaux réseaux contemporains sont ceux de téléphonie fixe, sans-fil cellulaires et Internet. NTT DoCoMo est le plus grand fournisseur au Japon, suivi par KDDI et Softbank, la majorité des téléphones fixes étant raccordés aux réseaux NTT West et NTT East. 
Les télécommunications ont favorisé la mise en place d'un système d'alerte aux tremblements de terre offrant aux utilisateurs un préavis de quelques secondes avant les premières secousses, leur permettant de se mettre à l'abri ou de fuir les immeubles. Dans un premier temps, la maintenance du réseau est essentielle pour cette première protection, puis lors des alertes de répliques éventuelles. Les séismes réguliers dans l'Archipel représentent autant d'occasions pour les opérateurs d'améliorer et de maintenir leurs réseaux respectifs durant des conditions dégradées. Ces opportunités antérieures d'apprentissage leur ont permis en 2011 de maintenir un niveau de performance global satisfaisant compte tenu de l'amplitude de la catastrophe, malgré des pannes importantes.

Les effets sur les réseaux de communication au-delà de la région du Tōhoku ont été limités. Leur utilisation, multipliée par 8 à 9 dans le cas de la région de Tokyo, n'a pas causé de problème critique contrairement à d'autres catastrophes, par exemple grâce à la mise en place d'une restriction d'appels et le transfert de la plupart des communications à un service d'informations sur les personnes touchées dans les zones sinistrées. La protection optimale de l'ensemble du réseau demeure très délicate, mais des efforts notables sur la redondance et la planification des interventions d'urgence sur le réseau physique ont montré des améliorations sensibles dans la reprise du système, en comparaison du tremblement de terre à Kobe en 1995. Ce dernier avait fourni en son temps de nombreuses leçons sur les possibilités d'amélioration de la résilience des télécommunications.

Un million et demi de lignes téléphoniques fixes sont tombées en panne entre le 11 et le 13 mars. Bien que la moitié d'entre elles aient été dépannées dès le 14 , il a fallu près de deux semaines pour rétablir $90 \%$ du service. Plus de la moitié des 1800 bâtiments de la NTT ont été touchés par le séisme ou le tsunami, la plupart par des pannes de courant. Ces derniers s'appuient en effet sur des batteries pouvant fonctionner de 24 à 48 heures, raison de la prolifération des pannes jusqu'au 13 mars. Les routes endommagées ont fait obstacle au déploiement rapide de groupes électrogènes portables pour recharger et prendre le relais des batteries. Des améliorations sur les conceptions sismiques souterraines abritant les câbles ont néanmoins rendu le réseau fixe plus robuste qu'en 1995 (Kwasinski et al. 2012).

Des pannes importantes ont affecté les équipements de communication sans fil, à nouveau en raison des pannes de courant multiples et des centres d'opérations endommagés par le tsunami. Les batteries des stations 
de base $\mathrm{e}^{53}$ sont encore plus vulnérables du fait de leur moindre autonomie d'environ huit heures, entraînant la mise hors service de milliers d'émetteurs dans la journée du 11 mars. Les opérateurs ont assuré une couverture d'urgence grâce à des centaines de téléphones satellitaires dans les zones les plus touchées, habituellement utilisés dans des régions isolées. Ils ont également ouvert leurs points d'accès $\mathrm{Wi}$ - Fi, en restreignant la bande passante aux communications de base. Les émetteurs ont peu à peu été remis en service mais plus d'un millier d'entre eux étaient toujours inopérants début avril. Dans ce contexte difficile, le succès de l'application de messagerie instantanée LINE s'explique par son recours à Internet, moins perturbé que les réseaux téléphoniques, pour communiquer ${ }^{54}$.

Une chute importante du nombre de recherches Internet a été notée au moment du séisme, sous l'effet là encore des pannes électriques, mais la situation est revenue à la normale au bout d'une heure. Le réseau a connu par la suite des pics à environ 200 fois le trafic normal. Étant composé d'un maillage dense, ce dernier a bien résisté en comparaison des coupures constatées sur ses homologues fixes et mobiles. Seule une petite partie de la vingtaine de câbles sous-marins reliant l'Archipel au reste du monde a été endommagée, occasionnant seulement une mise hors service de quelquesuns des milliers de centres de routage du trafic de l'Archipel. Plusieurs entreprises ont par ailleurs encouragé le télétravail afin d'éviter des déplacements trop contraignants à leurs employés.

Si les performances globales des différents réseaux de télécommunications sont restées convenables compte tenu du désastre, des sources d'amélioration sont apparues. Une compréhension plus fine des modes de défaillance et de planification des interventions d'urgence sont possibles pour développer un système encore plus résilient. Le déploiement du réseau de fibre optique en réponse à la demande croissante de vitesse de connexion et de volume de données transférées est déployé depuis lors, avec des installations antisismiques plus robustes. NTT a également annoncé investir 600 milliards de yens sur six ans dans des installations de production

53. Équipement sur site muni d'une antenne émettrice-réceptrice en communication avec les appareils mobiles.

54. "Chūōnippō » 中央日報 : https://japanese.joins.com/JArticle/149227 (dernière consultation le 26 juillet 2020). 
d'énergie solaire, éolienne et des systèmes de batteries pour les installations voisines essentielles, comme les hôpitaux et les usines en cas de panne de courant durant une catastrophe ou d'autres urgences ${ }^{55}$.

\section{Conclusion}

Cet article analyse la situation des transports et la réaction des autorités après le tremblement de terre et le tsunami survenus le 11 mars 2011, dont les conséquences désastreuses forment a posteriori une étape critique du développement d'infrastructures plus résilientes. De nombreux enseignements ont été tirés pour renforcer les capacités des infrastructures de transport. Le transport est par nature un domaine interdisciplinaire et cette situation de catastrophe n'a fait que mettre en valeur la nécessité d'une approche moins segmentée de leurs planifications et modèles respectifs. Si les études sur le développement durable se multiplient ces dernières années, celles sur le transport restent comparativement minces. Il s'en dégage toutefois quatre grandes catégories de recherches sur le transport durable (Badassa et al. 2020). La première concerne les définitions, les indicateurs et les paramètres de durabilité d'un système de transport durable; la deuxième se concentre sur l'évaluation des systèmes sociotechniques; la troisième se focalise sur les indicateurs de performance des transports urbains et la dernière se fonde sur l'analyse de la durabilité des villes et des services de transports interurbains.

La grande dépendance à l'automobile et au pétrole, résultante de l'étalement urbain continu dans l'Archipel, a été mise en évidence par les différentes pénuries, alimentant ainsi le débat sur la question de la durabilité urbaine au Japon et des konpakuto shiti コンパクトシティ (villes compactes, de l'anglais compact city). Elles ont dans un premier temps été abordées dans le contexte de la dépopulation du pays, avec notamment les genkai shüraku 限界集落 (villages seuils), des communautés en situation de dépeuplement

55. The Japan Times: https://www.japantimes.co.jp/news/2019/11/12/business/corporate-business/japans-ntt-600-billion-electricity (dernière consultation le 26 juillet 2020). 
avancé et risquant de disparaître lorsque plus de la moitié des habitants du village ont plus de 65 ans $^{56}$.

Autre sujet lié, la shōmetsu kanōsei jichitai 消滅可能性自治体 (possible extinction des collectivités territoriales locales) représente les centaines de collectivités répertoriées susceptibles de perdre plus de la moitié de leurs jeunes femmes et donc considérées en voie d'extinction ${ }^{57}$. Le gouvernement tente d'enrayer ce phénomène par la revitalisation régionale (chihō sōsei 地 方創生), une série de politiques visant à améliorer la vitalité de ces régions, notamment par de nouveaux moyens de transports. La stratégie des villes compactes date de bien avant 2011, mais la catastrophe a permis d'accélérer de nombreuses mesures dans le but de revitaliser les centres-villes ou d'apporter des améliorations aux centres urbains et aux réseaux de transports publics. La population du Japon étant susceptible de diminuer plus encore dans les prochaines décennies, l'enjeu de la réduction judicieuse de l'étalement urbain est urgente et celle-ci doit se faire en corrélation avec une planification plus prudente de l'utilisation des terres.

De nombreux endroits touchés par le tsunami ont connu des dégâts catastrophiques à plusieurs reprises par le passé. Beaucoup ont été reconstruits de manière similaire, en s'appuyant notamment sur plusieurs centaines de kilomètres de digues toujours plus hautes et robustes, sujettes à de nombreuses controverses, comme le sont aussi les relations entre le gouvernement et les sociétés de construction, parfois jugées incestueuses. La pertinence de ces digues est en effet sujette à débat, les perspectives techniques, économiques et sécuritaires devant être tempérées par les aspects sociaux, paysagers et écologiques. Cette décision est critiquée par beaucoup, qui clament la nécessité de ne pas répéter les erreurs passées de réinstallations dans des zones dangereuses, les zones résidentielles devant être placées à distance

56. Ōno Akira 大野晃 “Sanson no kōreika to genkai shūraku » 山村の高齢化と限界集落 (Vieillissement et implantations marginales dans les villages de montagne), 1991.

57. Masuda Hiroya 増田寛也, «Sutoppu «jinkō kyūgen shakai» : Kokumin no «kibō shusseiritsu» no jitsugen, chihō chūkaku kyoten toshi-ken no sōsei (kinkyū tokushū shōmetsu suru shichōson 523 : eshi suru chihō toshi » ストップ「人口急減社会」:国民の 「希望出生率」の実現、地方中核拠点都市圏の創成（緊急特集 消滅する市町村523:壊死する 地方都市) Stoppons le déclin de la population : réalisons le taux de natalité souhaité de la population et créons des centres régionaux (dossier spécial urgent : 523 municipalités en cours de disparition), 2014. 
et en hauteur. Les zones inondables, quant à elles, doivent être considérées comme des terres naturelles, des zones agricoles ou industrielles autour des ports de pêche.

Ces décisions reviennent souvent aux gouvernements locaux. Il est internationalement reconnu que la gouvernance locale représente la première ligne d'intervention en cas de catastrophe, le succès de la transition et de la reprise dépendant d'un fort leadership (Berke et al. 1993). Les agences nationales jouent un rôle important dans la préparation d'une catastrophe au travers de la mise en place de systèmes d'alertes, de plans d'évacuation et d'éducation continue, mais les gouvernements locaux demeurent les principaux responsables des normes de construction et de réglementations sur l'utilisation des sols, afin d'atténuer au possible les dégâts d'une catastrophe. Le Japon suit ce processus. Lors d'une catastrophe, le Premier ministre peut émettre des directives en tant que chef du Bōsai jōhō 防災 情報 (Centre de gestion des catastrophes au Japon, dépendant du MLIT); celles-ci découlent des saigai taisaku kihon hō 災害対策基本法 (Plan de base des contre-mesures en cas de catastrophe ${ }^{58}$ ) et sont révisées sur la base des leçons tirées du tremblement de terre de Kobe en 1995. Cependant, il n'existe pas d'entité nationale unique pour directement contrôler les mesures de prévention et de réaction aux catastrophes. Les responsables de ces organisations administratives, de gouvernements locaux et d'organismes publics, peuvent mener les actions jugées nécessaires en vertu de ces directives. Les conseils municipaux et départementaux de gestion des catastrophes sont tenus de préparer leurs plans respectifs tenant compte du contexte local sur le principe du plan de base des contre-mesures. En pratique, l'autorité du Premier ministre a peu de chances d'être exercée efficacement car les différentes organisations et gouvernements locaux ne font pas partie de la chaîne de commandement direct de ce dernier (Suzuki et al. 2013). Les interventions d'urgence, qui doivent en principe être menées par les autorités locales respectives, rendent le commandement central du Premier ministre difficile et lent.

Parmi les enseignements tirés au niveau national, le Comité pour la promotion de la gestion des catastrophes du conseil en charge de l'examen des

58. Centre de gestion des catastrophes du Japon : http://www.bousai.go.jp/taisaku/kihonhou/index.html (dernière consultation le 26 juillet 2020). 
mesures de prévention des catastrophes (Bōsai taisaku suishin kentō kaigi 防 災対策推進検討会議) propose d'intervenir sur trois points essentiels : examen des codes de conception des installations et préparation de manuels de réhabilitation; formulation de ces plans de réhabilitation durant des périodes normales sans catastrophe; renforcement de l'esprit de coopération entre gouvernement national et gouvernements locaux, promotion d'accords préalables avec les entreprises privées ${ }^{59}$. Ces trois points doivent pallier certains manquements notés durant la catastrophe de 2011, à savoir l'établissement de mécanismes d'arrangements financiers plus souples, la conclusion d'accords avec le secteur privé avant une catastrophe, la constitution d'équipes d'experts au niveau national, la construction d'infrastructures encore plus résistantes et permettre d'établir une priorité plus claire des infrastructures clés à réhabiliter. Le gouvernement national a également entrepris plusieurs mesures innovantes concernant le transport dans le cadre de la promotion des smart grids et smart communities, avec notamment une meilleure intégration des données topographiques, démographiques et sociales. Si les smart grids fonctionnent selon une approche descendante top-down, les smart communities proposent une approche montante bottom-up impliquant des citoyens, des organismes publics et des entreprises dans la construction de modes de vie moins énergivores et polluants pour une résilience à long terme accrue, mais ces smart grids peuvent également s'appliquer aux réponses de très court terme dans une situation d'urgence (Nakagawa et al. 2004). Différentes initiatives ont été lancées au cours du temps et promues par le ministère de l'Environnement (Kankyō daijin 環境大臣, MOE), le METI ou le MLIT. Citons notamment l'Eco-Town Model Project lancé en 1997, l'Environmetal Sustainable Transport Model Project (EST) en 2004, l'Eco-Model City Project (EMC) en 2008 ou encore Future Cities Initiatives en 2011 (Lecler et al. 2015).

Distinguer la réaction des autorités en trois phases distinctes - urgence, transition, reprise - met en exergue les défis à relever dans chaque phase (Nakanishi et al. 2011; Yoshida 2012). La phase d'urgence a nécessité une réparation immédiate des infrastructures et une capacité d'adaptation aux besoins en transport changeant sans cesse. Si la plupart des initiatives nationales ont montré quelques faiblesses, les interventions locales ont

59. Centre de gestion des catastrophes du Japon: http://www.bousai.go.jp/kaigirep/ chuobou/suishinkaigi/pdf/saishuu_hontai.pdf (dernière consultation le 26 juillet 2020). 
globalement bien fonctionné pour faire face aux problèmes prioritaires. Le sauvetage des personnes, le transport des fournitures d'urgence et la restauration des routes ont été réalisés à une vitesse inédite grâce à diverses initiatives locales s'appuyant sur des réseaux denses d'informations et d'entreprises. Les dirigeants locaux ont joué un rôle important dans la collecte des renseignements sur les différentes demandes en transport et dans l'acheminement des volontaires. La phase transitoire a montré l'importance de l'adaptabilité des services de transports afin de soutenir les individus dans la reprise d'une vie normale le plus rapidement possible. L'engagement communautaire et le capital social ont également été essentiels pour les prises de décisions dans un contexte anormal, complexe et de ressources limitées. La phase de reprise prélude la conception d'une communauté plus résiliente, capable d'anticiper plus efficacement la prochaine catastrophe en s'appuyant sur une interopérabilité et redondance accrue des infrastructures de transport.

Si le Japon souffre régulièrement de catastrophes naturelles, il accumule connaissances et technologies nécessaires pour les anticiper et s'y préparer. Le renforcement des capacités de résilience reste toutefois un défi, non seulement dans les zones soumises à des événements passés démontrant une mémoire parfois trop courte (Sato et al. 2017), mais encore et surtout pour les appliquer à échelle nationale, voire internationale. Il est également nécessaire de sensibiliser la population au fait que l'essentiel des dégâts et des morts durant une catastrophe est le résultat de la planification architecturale et urbaine. Au regard de la littérature existante, des recherches ultérieures sur la façon dont ces défis pourront être relevés dans la pratique seront cruciales pour étayer la planification d'infrastructures de transport résilientes (Faturechi et al. 2014). Sur le plus long terme, la catastrophe fait peut-être figure de violent rappel de la fragilité de nos infrastructures, qu'il est nécessaire de reconsidérer. En effet, si les chocs pétroliers ont occasionné une première transition énergétique contrainte et accélérée par des difficultés d'approvisionnement et par la flambée des prix à la suite des décisions politiques, le Japon a été poussé dans ses retranchements énergétiques le 11 mars 2011 par la réalité physique. S’il a réussi à prospérer économiquement, malgré de fortes contraintes énergétiques, géographiques et démographiques, la triple catastrophe lui a rappelé la grande corrélation entre énergie et économie, et elle a présenté une opportunité d'accélérer une transition énergétique sans doute salvatrice en vue du pic pétrolier mondial, des difficultés d'approvisionnement annoncées et du changement climatique. 


\section{Bibliographie}

Adger W. Neil 2000

«Social and Ecological Resilience: Are

They Related? ", Progress in Human

Geography, 24 (3) : 347-364.

AkAmatsu Takashi 赤松隆, NAGAE Takeshi 長江剛志, Ōsawa Minoru 大澤実 \& YAMAGUCH Hiromichi 山口裕通 2016 « Kyodai saigai-ji no gasorin busoku ni tai suru senryaku to sono shakai keizai hyōka : Higashi Nihon daishinsai ni okeru jisshō bunseki » 巨大災害時の ガソリン不足に対する戦略とその社会経 済評価:東日本大震災における実証分析 (Stratégies de distribution d'essence suite à un désastre pour réduire les pertes socioéconomiques, leçons du séisme de 2011 de la côte Pacifique du Tōhoku), Journal of Japan Society of Civil Engineers, Ser. D3 (Infrastructure Planning and Management), 72 (5) : 1-19.

Aokı Masahiko \& Rothwel Geoffrey 2013 «A Comparative Institutional Analysis of the Fukushima Nuclear Disaster: Lessons and Policy Implications », Energy Policy, $53: 240-247$.

\section{Aratani Tarō 荒谷太郎 et al. 2013a} « Higashi Nihon daishinsai-ji no kōkūki katsudō to kūkō un.yō no jittai bunseki. Iwate Hanamaki, Yamagata, Fukushima kūkō o taishō to shite » 東日本大震災 時の航空機活動と空港運用の実態分析一 いわて花巻·山形·福島空港を対象とし て- (Activités aériennes et opérations aéroportuaires après le séisme de 2011 de la côte Pacifique du Tōhoku.
Aéroports d'Iwate, Hanamaki, Yamagata et Fukushima), Journal of Japan Society of Civil Engineers, Ser. D3 (Infrastructure Planning and Management), 69 (5) : 229-246.

Aratani Tarō et al. 2013b "Lessons and Challenges in Airport Operation During a Disaster: Case Studies on Iwate Hanamaki Airport, Yamagata Airport, and Fukushima Airport During the Great East Japan Earthquake ", Journal of JSCE, 1 (1) : 286-297.

\section{BadAssa Bayissa Badada, Sun Baiqing \&} QıAO Lixin 2020

«Sustainable Transport Infrastructure and Economic Returns: A Bibliometric and Visualization Analysis ", Sustainability, 12 (5) : 2033.

\section{Barker Kash, Hosseini Seyedmohsen \&} Ramirez-Marquez Jose E. 2016 «A Review of Definitions and Measures of System Resilience ", Reliability Engineering \& System Safety, 145 : 47-61.

Berke Philip, Kartez Jack \&

Wenger Dennis 1993

«Recovery After Disaster: Achieving Sustainable Development, Mitigation and Equity », Disasters, 17 (2), 93-109.

\section{BLaCK John, NaKanishi Hitomi \&} Matsuo Kojiro 2014 « Disaster Resilience in Transportation: Japan Earthquake and Tsunami 2011 », International Journal of Disaster Resilience in the Built Environment, 5 (4) : 341-361. 
Christopher Martin \& Peck Helen 2004 «Building the Resilient Supply Chain », International Journal of Logistics Management, 15 (2) : 1-13.

Faturechi Reza \& Miller-Hooks Elise 2014 «Measuring the Performance of Transportation Infrastructure Systems in Disasters: A Comprehensive Review », Journal of Infrastructure Systems, 21 (1) : 04014025.

Fusıмото Kosuke, Ishikawa Fumio, Iwata Michitoshi \& TATSUKI Shinichi 2018 «Restoration of Joban Line Railway Facilities Damaged by Tōhoku-Pacific Ocean Earthquake ", in HoRDIJk Dick A. \& Luković Mladena, High Tech Concrete. Where Technology and Engineering Meet, Cham, Springer : 1942-1950.

\section{Fusu Makoto 藤生慎 et al. 2011} « 2011 nen Tōhoku chihō Taiheiyō oki jishin ni okeru Sanriku tetsudō no higai chōsa sokuhō »2011年東北地方太平 洋沖地震における三陸鉄道の被害調査速 報 (Premier rapport sur l'enquête des dommages subis par Sanriku Railway lors du séisme de 2011 de la côte Pacifique du Tōhoku), The University of Tokyo, Institute of Industrial Science, Seisan kenkyū, 63 (4) : 535-540.

\section{Fuנlu Makoto et al. 2012}

«Tōhoku chihō Taiheiyō oki jishin ni yoru kitaku kon.nansha no idō kaishi jikoku ni kansuru bunseki » 東北地方太平洋沖地 震による帰宅困難者の移動開始時刻に関す る分析 (Analyse du comportement des personnes ayant des difficultés à rentrer chez elles dans la région métropolitaine de Tokyo lors du séisme de 2011 de la côte Pacifique du Tōhoku), Journal of Japan Association for Earthquake Engineering, 12 (5) : 180-191.

Graham Stephen \& Thrift Nigel 2007 «Understanding repair and maintenance », Theory, Culture \& Society, 24 (3) : 1-25.

\section{Granier Benoit 2019}

«Gouverner la consommation d'énergie des ménages. Renouvellement des enjeux et des instruments d'intervention (1973-2017) », Ebisu. Études japonaises, 56 (Consommer au Japon, consommer le Japon) : 223-252.

https://journals.openedition.org/ ebisu/4560 (dernière consultation le 28 janvier 2021).

\section{HaYASH Yoshinari 林能成 2012}

« Higashi Nihon daishinsai ni okeru tetsudō no hinan yūdō » 東日本大震災 における鉄道の避難誘導 (Processus d'évacuation des passagers des trains lors du séisme de 2011 de la côte Pacifique du Tōhoku), Journal of Societal Safety Sciences, 2 : 36-37.

\section{HirakaWa Yuta 平川雄太 et al. 2017} «Saigai denshō wa tsunami hinan kōdō o yūin shita no ka. Rikuzentakadashi ni okeru shitsumonshi chōsa o mochiita jirei bunseki » 災害伝承は津波避難 行動を誘引したのかー陸前高田市にお ける質問紙調査を用いた事例分析一 (Les avertissements traditionnels de catastrophe ont-ils favorisé l'évacuation durant le tsunami? Étude de cas par questionnaire dans la ville de 
Rikuzentakata, département d'Iwate), Journal of Social Safety Science, 31 : 69-76.

Holuing Crawford 1973

« Resilience and Stability of Ecological Systems ", Annual Review of Ecology and Systematics, 4 (1) : 1-23.

Hollnagel Erik, Leveson Nancy \& Woods David D. 2006

Resilience Engineering: Concepts and Precepts, Farnham, Ashgate Publishing.

\section{Kajtani Yoshio, Chang Stephanie \&}

TAtano Hirokazu 2013

« Economic Impacts of the 2011

Tohoku-Oki Earthquake and Tsunami », Earthquake Spectra, 29 (1 suppl) : 457-478.

\section{KAZAMA Motoki 風間基樹 2012} « 2011 nen Tōhoku chihō Taiheiyō oki jishin higai no gaiyō to jiban kōgakuteki kadai » 2011 年東北地方太平洋沖地震被害 の概要と地盤工学的課題 (Vue d'ensemble des dommages et des problèmes géotechniques du séisme de 2011 de la côte Pacifique du Tōhoku), Japanese Geotechnical Journal, 7 (1) : 1-11.

\section{Kazama Motoki \& Noda Toshihiro 2012}

«Damage Statistics (Summary of the 2011 off the Pacific Coast of Tōhoku Earthquake damage) », Soils and Foundations, 52 (5) : 780-792.

Kırota Yūtarō 清田裕太郎, IwaKURA Seiji 岩倉成志, NonAKA Yasuhiro 野中康弘 2014 "Higashi Nihon daishinsai-ji no guriddorokku genshō ni motozuku tokunai dōro no botorunekku kasho no kōsatsu » 東日本大震災時のグリッ ドロック現象に基づく都区内道路のボト ルネック箇所の考察 (Gridlocks urbains et goulots d'étranglement du réseau routier de Tokyo suite au séisme de 2011 de la côte Pacifique du Tōhoku), Journal of Japan Society of Civil Engineers, Ser. D3 (Infrastructure Planning and Management), 70 (5) : 1059-1066.

\section{Kumagal Kentaro 2013}

«Tsunami-induced Debris of Freight Containers due to the 2011 off the Pacific Coast of Tōhoku Earthquake ", Journal of Disaster FactSheets : 1-25.

Kwasinski Alexis \& TAng Alex K. 2011 «Telecommunications Performance in the $M=9.0$ Off-shore East Coast of Japan Earthquake and Tsunami March 11 2011 ", Proceedings of the International Symposium on Engineering Lessons Learned from the Giant Earthquake : 1514-1525.

LeCLer Yveline \& FaIVRe D'ARCIer Bruno 2015 «Smart Cities Experiments in France and Japan: Preparing the Energy Transition ", Association of Asian Studies Annual Conference, AAS, Chicago, United States.

Nakagawa Yuko \& Shaw Rajib 2004 «Social Capital: A Missing Link to Disaster Recovery », International Journal of Mass Emergencies and Disasters, 22 (1), 5-34.

Reghezza-Zitt Magali \& Rufat Samuel 2015 « Resilience Imperative: Uncertainty, Risks and Disasters », Elsevier. 


\section{Rose Adam 2004}

«Defining and Measuring Economic

Resilience to Disasters », Disaster

Prevention and Management: An

International Journal, 13 (4) : 307-314.

\section{SAmUels Richard J. 2013}

3.11: Disaster and Change in Japan, Ithaca, Cornell University Press.

\section{SheffI Yossi 2005}

The Resilient Enterprise: Overcoming Vulnerability for Competitive Advantage, Cambridge, MIT Press.

\section{Suzuki Itoko \& Kaneko Yuko 2013}

Japan's Disaster Governance: How was the 3.11 Crisis Managed?, Berlin, Springer Science \& Business Media.

WILson Rockie K. 2012

«Operation Tomodachi: A Model for American Disaster Response Efforts and the Collective Use of Military Forces Abroad », John F Kennedy School of Government Cambridge, Harvard University.

\section{YosHiDA Itsuki 吉田樹 2012}

« Higashi Nihon daishinsai hisaichi ni okeru mobiriti to hinansha no akuseshibiriti ni kansuru kōsatsu (shō tokushū Daikibo saigai-ji no mobiriti kakuho) » 東日本大震災被災地におけるモビ リティと避難者のアクセシビリティに関する考 察(小特集 大規模災害時のモビリティ確保) (Étude sur la mobilité et l'amélioration de l'accessibilité individuelle dans une zone sinistrée suite au séisme de 2011 de la côte Pacifique du Tōhoku), Traffic Science, 43 (1) : 11-18. 